\title{
Measurements of cross sections and decay properties of the isotopes of elements 112, 114, and 116 produced in the fusion reactions ${ }^{233,238} \mathrm{U},{ }^{242} \mathrm{Pu}$, and ${ }^{248} \mathrm{Cm}+{ }^{48} \mathrm{Ca}$
}

Yu. Ts. Oganessian, V. K. Utyonkov, Yu. V. Lobanov, F. Sh. Abdullin, A. N. Polyakov, I. V. Shirokovsky, Yu. S. Tsyganov, G. G. Gulbekian, S. L. Bogomolov, B. N. Gikal, A. N. Mezentsev, S. Iliev, V. G. Subbotin, A. M. Sukhov, A. A. Voinov, G. V. Buklanov, K. Subotic, V. I. Zagrebaev, and M. G. Itkis

Joint Institute for Nuclear Research, 141980 Dubna, Russian Federation

\author{
J. B. Patin, K. J. Moody, J. F. Wild, M. A. Stoyer, N. J. Stoyer, D. A. Shaughnessy, J. M. Kenneally, \\ P. A. Wilk, and R. W. Lougheed \\ Lawrence Livermore National Laboratory, University of California, Livermore, California 94551, USA
}

\author{
R. I. Il'kaev and S. P. Vesnovskii \\ Russian Federal Nuclear Center, All-Russian Research Institute of Experimental Physics, 607190 Sarov, Russian Federation
}

(Received 9 August 2004; published 17 December 2004; corrected 28 January 2005)

\begin{abstract}
We have studied the dependence of the production cross sections of the isotopes ${ }^{282,283} 112$ and ${ }^{286,287} 114$ on the excitation energy of the compound nuclei ${ }^{286} 112$ and ${ }^{290} 114$. The maximum cross section values of the $x n$-evaporation channels for the reaction ${ }^{238} \mathrm{U}\left({ }^{48} \mathrm{Ca}, x n\right){ }^{286-x} 112$ were measured to be $\sigma_{3 n}=2.5_{-1.1}^{+1.8} \mathrm{pb}$ and $\sigma_{4 n}=0.6_{-0.5}^{+1.6} \mathrm{pb}$; for the reaction ${ }^{242} \mathrm{Pu}\left({ }^{48} \mathrm{Ca}, x n\right)^{290-x} 114: \sigma_{2 n} \sim 0.5 \mathrm{pb}, \sigma_{3 n}=3.6_{-1.7}^{+3.4} \mathrm{pb}$, and $\sigma_{4 n}=4.5_{-1.9}^{+3.6} \mathrm{pb}$. In the reaction ${ }^{233} \mathrm{U}\left({ }^{48} \mathrm{Ca}, 2-4 n\right){ }^{277-279} 112$ at $E^{*}=34.9 \pm 2.2 \mathrm{MeV}$ we measured an upper cross section limit of $\sigma_{x n} \leqslant 0.6 \mathrm{pb}$. The observed shift of the excitation energy associated with the maximum sum evaporation residue cross section $\sigma_{\mathrm{ER}}\left(E^{*}\right)$ to values significantly higher than that associated with the calculated Coulomb barrier can be caused by the orientation of the deformed target nucleus in the entrance channel of the reaction. An increase of $\sigma_{\mathrm{ER}}$ in the reactions of actinide targets with ${ }^{48} \mathrm{Ca}$ is consistent with the expected increase of the survivability of the excited compound nucleus upon closer approach to the closed neutron shell $N=184$. In the present work we detected 33 decay chains arising in the decay of the known nuclei ${ }^{282} 112,{ }^{283} 112,{ }^{286} 114$, ${ }^{287} 114$, and ${ }^{288} 114$. In the decay of ${ }^{287} 114(\alpha) \rightarrow{ }^{283} 112(\alpha) \rightarrow{ }^{279} 110(\mathrm{SF})$, in two cases out of 22 , we observed decay chains of four and five sequential $\alpha$ transitions that end in spontaneous fission of ${ }^{271} \mathrm{Sg}\left(T_{\alpha / \mathrm{SF}}\right.$ $\left.=2.4_{-1.0}^{+4.3} \mathrm{~min}\right)$ and ${ }^{267} \operatorname{Rf}\left(T_{\mathrm{SF}} \sim 2.3 \mathrm{~h}\right)$, longer decay chains than reported previously. We observed the new nuclide ${ }^{292} 116\left(T_{\alpha}=18_{-6}^{+16} \mathrm{~ms}, E_{\alpha}=10.66 \pm 0.07 \mathrm{MeV}\right)$ in the irradiation of the ${ }^{248} \mathrm{Cm}$ target at a higher energy than in previous experiments. The observed nuclear decay properties of the nuclides with $Z=104-118$ are compared with theoretical nuclear mass calculations and the systematic trends of spontaneous fission properties. As a whole, they give a consistent pattern of decay of the 18 even- $Z$ neutron-rich nuclides with $Z$ $=104-118$ and $N=163-177$. The experiments were performed with the heavy-ion beam delivered by the U400 cyclotron of the FLNR (JINR, Dubna) employing the Dubna gas-filled recoil separator.
\end{abstract}

DOI: 10.1103/PhysRevC.70.064609 PACS number(s): 25.70.Gh, 23.60.+e, 25.85.Ca, 27.90.+b

\section{INTRODUCTION}

According to the microscopic nuclear models, the limits of the existence of the heavy nuclei, as well as their decay properties, are completely determined by nuclear shell effects. For the heaviest (superheavy) elements in the vicinity of the hypothetical closed spherical shells $Z=114$ (or possibly 120,122 , or 126 ) and $N=184$, the increase of nuclear binding energy results in a considerable increase of stability with respect to various decay modes: $\alpha$ decay or spontaneous fission (SF). We may also speculate that the high fission barriers of the superheavy nuclei in their ground states may persist at low excitation energies, resulting in an increase in their production cross sections, or to be more precise their survival probability in the process of deexcitation of the compound nucleus that is produced in the complete fusion of massive nuclei.

Both the production cross sections and the stability of superheavy nuclides are expected to increase on closer ap- proach to the neutron shell $N=184$. Therefore, our first experiments aimed at the synthesis of the heaviest nuclei involved the complete fusion reactions ${ }^{244} \mathrm{Pu},{ }^{248} \mathrm{Cm}+{ }^{48} \mathrm{Ca}$ that lead to the compound nuclei ${ }^{292} 114(N=178)$ and ${ }^{296} 116$ $(N=180)$ with the maximum accessible neutron numbers $[1,2]$.

At the same time, it has been difficult to explore the mechanism of fusion of ${ }^{48} \mathrm{Ca}$ projectiles with actinide targets because of the extremely low yield of evaporation residues (ERs). Following the successful completion of the experiments in which fission in ${ }^{48} \mathrm{Ca}$-induced reactions was studied [3] we decided to investigate the survival probabilities of the compound nuclei by measuring excitation functions for producing evaporation residues. The first such measurements were performed using the reaction ${ }^{244} \mathrm{Pu}\left({ }^{48} \mathrm{Ca}, 3-5 n\right){ }^{287-289} 114$ [4]. In the present work, we further develop these investigations with different targets ${ }^{233} \mathrm{U}$, ${ }^{238} \mathrm{U}$, and ${ }^{242} \mathrm{Pu}$, producing compound nuclei with $Z=112$ and 114 that have fission barriers and neutron binding ener- 
gies different from those of ${ }^{292} 114$. We also present the results of the cross section measurements of the reaction ${ }^{248} \mathrm{Cm}+{ }^{48} \mathrm{Ca}$ at the excitation energies $E^{*}=30.5$ and $33.0 \mathrm{MeV}[2]$ and recent experiments at $E^{*}=38.9 \mathrm{MeV}$. In the future the measurement of the excitation function of this reaction will be continued at higher bombarding energy.

In the reactions ${ }^{242} \mathrm{Pu},{ }^{248} \mathrm{Cm}+{ }^{48} \mathrm{Ca}$, in the $4 n$ evaporation channel, we would expect the production of three even-even nuclides, ${ }^{282} 112(N=170),{ }^{286} 114(N=172)$, and ${ }^{292} 116(N$ $=176$ ). Comparing their radioactive decay properties with the available data on previously synthesized even-even ${ }^{284} 112,{ }^{288} 114$, and ${ }^{290} 116$ would then define the decay properties of these even-even isotopes as a function of neutron number and for their odd-mass neighbors as well. One should note also that the isotopes ${ }^{282} 112,{ }^{286} 114$, and ${ }^{290} 116$ [4] are contained in the $\alpha$-decay chain of the isotope ${ }^{294} 118$ that we previously observed in the reaction ${ }^{249} \mathrm{Ca}\left({ }^{48} \mathrm{Ca}, 3 n\right)$ [5]. This reaction is to be studied in more detail in future experiments. In addition, we also intended to check the decay data for the isotopes ${ }^{283} 112$ and ${ }^{287} 114$ (the $3 n$-channel products of the reactions ${ }^{238} \mathrm{U},{ }^{242} \mathrm{Pu}+{ }^{48} \mathrm{Ca}$ ) that were observed in experiments with the VASSILISSA separator in 1998-2003 [6,7].

Finally, reactions that produce nuclides with $Z=112$ and $N \leqslant 166$ make it possible to study the production and properties of deformed nuclei in the vicinity of the closed neutron shell $N=162$, which have been produced in cold fusion reactions [8]. In the reaction ${ }^{233} \mathrm{U}+{ }^{48} \mathrm{Ca}$, the $4 n$-evaporation channel leads to ${ }^{277} 112$, synthesized in the cold fusion reaction ${ }^{208} \mathrm{~Pb}\left({ }^{70} \mathrm{Zn}, n\right){ }^{277} 112$ [9]; the $\alpha$-decay of the $3 n$ evaporation, isotope ${ }^{278} 112$, leads to the known nuclei ${ }^{270} \mathrm{Hs}$ and ${ }^{266} \mathrm{Sg}$, previously produced in the hot fusion reactions ${ }^{248} \mathrm{Cm}\left({ }^{26} \mathrm{Mg}, 4 n\right){ }^{270} \mathrm{Hs}[10]$ and ${ }^{248} \mathrm{Cm}\left({ }^{22} \mathrm{Ne}, 4 n\right){ }^{266} \mathrm{Sg}$ [11], respectively.

\section{EXPERIMENTAL TECHNIQUE}

The ${ }^{48} \mathrm{Ca}$-ion beam was accelerated by the U400 cyclotron at the Flerov Laboratory of Nuclear Reactions. The typical beam intensity at the target was $1.2 p \mu \mathrm{A}$. The $32-\mathrm{cm}^{2}$ rotating targets consisted of the enriched isotopes ${ }^{233} \mathrm{U}$ (99.97\%), ${ }^{238} \mathrm{U}(99.3 \%),{ }^{242} \mathrm{Pu}(99.98 \%)$, and ${ }^{248} \mathrm{Cm}(97.4 \%)$ deposited as $\mathrm{U}_{3} \mathrm{O}_{8}, \mathrm{PuO}_{2}$, and $\mathrm{CmO}_{2}$ onto 1.5- $\mu \mathrm{m}$ Ti foils to thicknesses of about $0.44,0.35,0.40$, and $0.35 \mathrm{mg} \mathrm{cm}^{-2}$, respectively.

The ERs recoiling from the target were separated in flight from ${ }^{48} \mathrm{Ca}$ beam ions, scattered particles, and transferreaction products by the Dubna gas-filled recoil separator [12]. The transmission efficiency of the separator for $Z$ $=112$ and 114 nuclei is estimated to be approximately $40 \%$ [12]. ERs passed through a time-of-flight (TOF) system (with detection efficiency of 99.9\%) and were implanted in a $4 \times 12 \mathrm{~cm}^{2}$ semiconductor detector array with 12 vertical position-sensitive strips, located in the separator's focal plane. This detector was surrounded by eight $4 \times 4 \mathrm{~cm}^{2}$ side detectors without position sensitivity, forming a box open to the front (beam) side. The position-averaged detection efficiency for $\alpha$ decays of implanted nuclei was $87 \%$ of $4 \pi$. The detection system was tested by registering the recoil nuclei and decays ( $\alpha$ or SF) of the known isotopes of No and Th, as well as their descendants, produced in the reactions ${ }^{206} \mathrm{~Pb}\left({ }^{48} \mathrm{Ca}, x n\right)$, and ${ }^{\mathrm{nat}} \mathrm{Yb}\left({ }^{48} \mathrm{Ca}, x n\right)$, respectively. The energy resolution for $\alpha$ particles absorbed in the focal-plane detector was $55-95 \mathrm{keV}$. The $\alpha$ particles that escaped the focal-plane detector at different angles and registered in a side detector had an energy resolution of the summed signals (side detector plus residual focal-plane detector) of $140-220 \mathrm{keV}$. If an $\alpha$ particle was detected only by a side detector (and thus its position was lost) the uncertainty in determining its energy increased to $450-500 \mathrm{keV}$; its assignment to an observed decay chain is determined from the calculated probability of random correlations based on the decay rate in the side detectors associated with the actual experimental conditions.

The full width at half maximum (FWHM) position resolutions of the signals of correlated decays of nuclei implanted in the detectors were $0.9-1.4 \mathrm{~mm}$ for ER- $\alpha$ signals and $0.5-0.9 \mathrm{~mm}$ for ER-SF signals. It can be seen from the data presented below that all the correlated events observed in these experiments were registered with position deviations comparable to the given position resolutions. Fission fragments from the decay of ${ }^{252}$ No implants produced in the ${ }^{206} \mathrm{~Pb}+{ }^{48} \mathrm{Ca}$ reaction were used for the total kinetic energy (TKE) calibration. The measured fragment energies were not corrected for the pulse-height defect of the detectors, or for energy loss in the detectors' entrance windows, dead layers, and the pentane gas filling the detection system. The mean sum energy loss of fission fragments from the SF decay of ${ }^{252}$ No was about $20 \mathrm{MeV}$. For the estimation of the total kinetic energy of fissioning nuclei the measured fragment energies were increased by this value [see Fig. 6(a) below]. The systematic uncertainty in estimating the TKE value is about $5 \mathrm{MeV}$.

From model calculations and the available experimental data for neighboring nuclei one can estimate the expected $\alpha$-particle energies for the isotopes of elements 112 and 114 produced in the reactions under study. This allowed us to employ a special low-background detection scheme for the investigated nuclides [2,4]. In the bombardment of ${ }^{242} \mathrm{Pu}$, the beam was switched off after a recoil signal was detected with parameters of implantation energy and TOF expected for $Z$ $=114$ evaporation residues, followed by an $\alpha$-like signal with an energy of $9.9 \leqslant E_{\alpha} \leqslant 10.35 \mathrm{MeV}$, in the same strip, within a (1.4-1.9)-mm-wide position window and a time interval of $\Delta t=4 \mathrm{~s}$. During approximately $20 \%$ of the duration of the experiment, additional energy and time intervals were set to switch the beam off following the detection of a second $\alpha$ transition (for those cases if the first $\alpha$ particle was not registered with a position-correlated signal to the preceding ER): $9.48 \leqslant E_{\alpha} \leqslant 9.75 \mathrm{MeV}, \Delta t=10 \mathrm{~s}$. The duration of the pause in the beam was determined from the observed pattern of out-of-beam $\alpha$ decays and varied from 1 to $12 \mathrm{~min}$. Thus, all the expected sequential decays of the daughter nuclides with $Z \leqslant 112$ were expected to be observed in the absence of beam-associated background. In the experiments ${ }^{238} \mathrm{U}+{ }^{48} \mathrm{Ca}$ and ${ }^{233} \mathrm{U}+{ }^{48} \mathrm{Ca}$, the energy and time intervals were 9.43 $\leqslant E_{\alpha} \leqslant 9.63 \mathrm{MeV}, \Delta t=12 \mathrm{~s}$ and $10.3 \leqslant E_{\alpha} \leqslant 11.8 \mathrm{MeV}, \Delta t$ $=0.3 \mathrm{~s}$, respectively. In the ${ }^{248} \mathrm{Cm}+{ }^{48} \mathrm{Ca}$ experiment, the energy and time intervals were $10.4 \leqslant E_{\alpha} \leqslant 10.8 \mathrm{MeV}, \Delta t$ $=0.2 \mathrm{~s}$ for the first $\alpha$ particle and $9.7 \leqslant E_{\alpha} \leqslant 9.94 \mathrm{MeV}, \Delta t$ $=2 \mathrm{~s}$ for the second $\alpha$ particle. 
TABLE I. Reaction-specific lab-frame beam energies in the middle of the target layers used in the present work, corresponding excitation energies [13], and total beam doses for the given reactions.

\begin{tabular}{cccc}
\hline \hline Reaction & $E_{\text {beam }}(\mathrm{MeV})$ & $E^{*}(\mathrm{MeV})$ & Beam dose \\
\hline${ }^{242} \mathrm{Pu}+{ }^{48} \mathrm{Ca}$ & 235 & $30.4-34.7$ & $5.0 \times 10^{18}$ \\
& 238 & $33.1-37.4$ & $4.9 \times 10^{18}$ \\
& 244 & $38.0-42.4$ & $4.7 \times 10^{18}$ \\
& 250 & $43.0-47.2$ & $3.2 \times 10^{18}$ \\
${ }^{238} \mathrm{U}+{ }^{48} \mathrm{Ca}$ & 230 & $29.3-33.5$ & $5.8 \times 10^{18}$ \\
& 234 & $32.9-37.2$ & $7.1 \times 10^{18}$ \\
${ }^{233} \mathrm{U}+{ }^{48} \mathrm{Ca}$ & 240 & $37.7-41.9$ & $5.2 \times 10^{18}$ \\
${ }^{248} \mathrm{Cm}+{ }^{48} \mathrm{Ca}$ & 240 & $32.7-37.1$ & $7.7 \times 10^{18}$ \\
\hline \hline
\end{tabular}

\section{EXPERIMENTAL RESULTS}

Irradiations of the ${ }^{242} \mathrm{Pu},{ }^{238} \mathrm{U},{ }^{233} \mathrm{U}$, and ${ }^{248} \mathrm{Cm}$ targets by ${ }^{48} \mathrm{Ca}$ projectiles at various energies were performed in September-November, 2003; December, 2003-February, 2004; April, 2004; and April-May, 2004; respectively; the corresponding integrated, on-target beam doses of 1.8 $\times 10^{19}, 1.8 \times 10^{19}, 0.8 \times 10^{19}$, and $0.7 \times 10^{19}$ ions were accumulated. Experimental conditions are summarized in Table I. Excitation energies of the compound nuclei at given ion energies are calculated using the masses of [13], taking into account the thickness of the targets and the energy spread of the incident cyclotron beam $(\approx \pm 1 \%)$. The beam energy losses in the separator's entrance window and target backing (both 1.5- $\mu \mathrm{m}$ Ti foils) and target layer were calculated using available data of Hubert et al. or Northcliffe and Schilling in other cases [14].

The spectrum of $\alpha$-like signals (all events without TOF signal) in all strips in the energy range of $6 \leqslant E_{\alpha} \leqslant 10 \mathrm{MeV}$ accumulated over the whole series of ${ }^{242} \mathrm{Pu}+{ }^{48} \mathrm{Ca}$ experiments is shown in Fig. 1(a). In the figure, we also show the $\alpha$-particle spectrum detected in beam-off time intervals (see above). In the beam-off $\alpha$-particle spectrum we observe the peaks originating from $\alpha$-decaying isotopes of Po, the decay products of the long-lived isotopes of $\mathrm{Ra}$-Th produced in transfer reactions. Note that in the high-energy part of the $\alpha$-particle spectrum $\left(E_{\alpha} \geqslant 9.3 \mathrm{MeV}\right)$ only 22 events were detected, six of them (marked black), as we will demonstrate below, belonging to the decay chains of ${ }^{287} 114$.

The total spectrum of high-energy signals with $E$ $\geqslant 50 \mathrm{MeV}$ (without TOF signal) is presented in Fig. 1(b). In cases when fission signals were registered by both the focalplane and the side detector, the sum energy is given. The background signals (part of the scattered ${ }^{48} \mathrm{Ca}$ ions, fragments of the induced fission of the target, etc.) appear in the energy range $E \leqslant 130 \mathrm{MeV}$; they are not observed in the beam-off spectrum. The signals of SF fragments arising in the decays of heavy nuclei are expected at higher energies, with $E \geqslant 130 \mathrm{MeV}$.

During the entire $1120-\mathrm{h}{ }^{242} \mathrm{Pu}+{ }^{48} \mathrm{Ca}$ run, with a total beam dose of $1.8 \times 10^{19}$, we detected 55 fissionlike signals
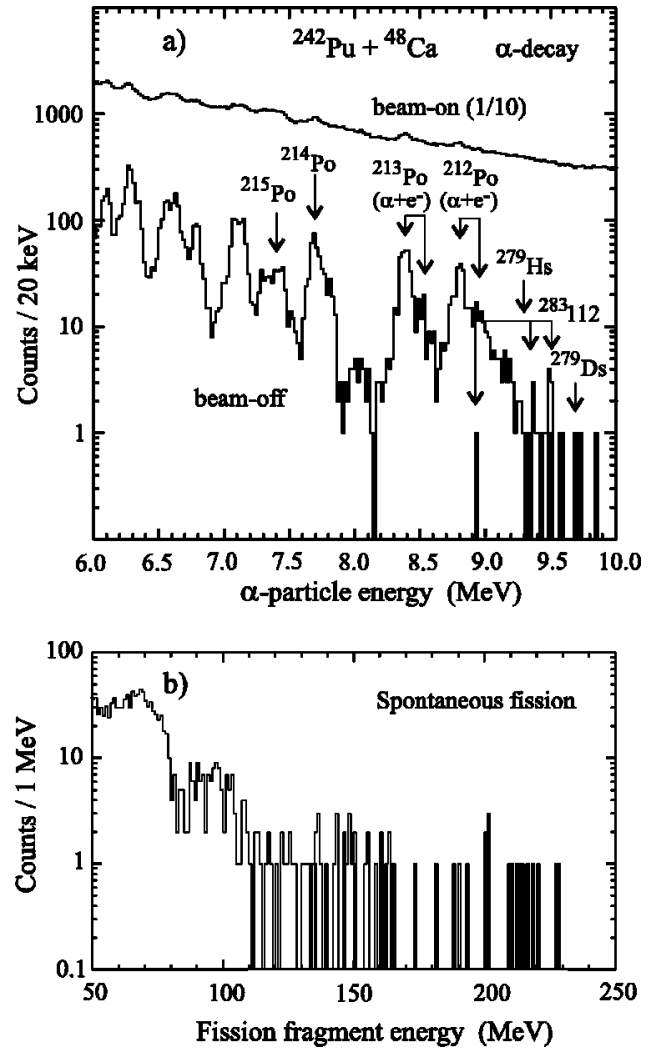

FIG. 1. (a) Total beam-on and beam-off $\alpha$-particle energy spectra of events registered by the focal-plane detector and by both the focal-plane and side detectors in the reaction ${ }^{242} \mathrm{Pu}+{ }^{48} \mathrm{Ca}$. The solid histogram shows the energies of events observed during beam-off periods in the correlated decay chains (see Fig. 2 and Table II). (b) Total fission-fragment energy spectrum; the solid histogram shows the energies of events observed in the correlated decay chains.

with $E_{\mathrm{F}} \geqslant 130 \mathrm{MeV}$ [Fig. 1(b)]. Eight events in the energy interval $E_{\mathrm{F}}=140-165 \mathrm{MeV}$ can be attributed to the SF decay of the well-known isomer ${ }^{242 \mathrm{mf}} \mathrm{Am}\left(T_{\mathrm{SF}}=14 \mathrm{~ms}\right)$ produced in transfer reactions. SF from long-lived nuclei, e.g., ${ }^{252} \mathrm{Cf}$ $\left(T_{1 / 2}=2.65 \mathrm{yr}\right)$, accumulated in previous experiments $[4,5,15]$, could be expected to contribute about half of the remaining 47 events. The 25 events that we have assigned to the decay chains of element 114 have been marked in black; see Fig. 1(b). The strip-averaged probability to detect random ER-like signals with $E_{\mathrm{ER}}=6.5-14.5 \mathrm{MeV}$ within a position window of $2 \mathrm{~mm}$ and time interval of $1 \mathrm{~s}$ was about $2.5 \times 10^{-3}$. Thus, the ER-SF sequences attributed to the decays of element 114 were separated from ${ }^{242 \mathrm{mf}} \mathrm{Am}$ and background long-lived nuclei by the high energy of fission fragments and/or ER-SF time intervals corresponding to the halflife of the mother isotope determined from ER- $\alpha$-SF decay chains (see Table II below).

Note that, due to the low number of signals with $E$ $\geqslant 130 \mathrm{MeV}$, there is no advantage in detecting spontaneous fission with the beam off.

In the entire series of ${ }^{242} \mathrm{Pu}+{ }^{48} \mathrm{Ca}$ experiments, we had about 3990 beam interrupts lasting a total of $82 \mathrm{~h}$. The total spectrum of $\alpha$ particles that caused the beam to be switched off is shown in Fig. 2. Here, the solid histogram shows the energies of events that caused the beam to be switched off 
TABLE II. Decay chains observed in the ${ }^{242} \mathrm{Pu}+{ }^{48} \mathrm{Ca}$ reaction. Bold events were registered during a beam-off period. Time intervals for events following missing $\alpha$ were measured from preceding registered events and are shown in italic. The $\alpha$-particle energy errors are shown in parentheses.

\begin{tabular}{|c|c|c|c|c|c|c|c|c|}
\hline \multicolumn{9}{|c|}{ (a) ER- $\alpha$-SF chain $\left({ }^{288} 114\right)$} \\
\hline $\begin{array}{c}E_{\mathrm{Lab}} \\
(\mathrm{MeV})\end{array}$ & Strip & $\begin{array}{c}E_{\mathrm{ER}} \\
(\mathrm{MeV})\end{array}$ & $\begin{array}{c}\delta t_{\text {ER- } \alpha} \\
\text { (s) }\end{array}$ & $\begin{array}{c}E_{\alpha} \\
(\mathrm{MeV})\end{array}$ & $\begin{array}{c}\delta t_{\alpha-\mathrm{SF}} \\
\quad(\mathrm{s})\end{array}$ & $\begin{array}{c}E_{\mathrm{SF}} \\
(\mathrm{MeV})\end{array}$ & & \\
\hline 235 & 9 & 9.5 & 3.852 & $9.86(45)^{\mathrm{a}}$ & 0.202 & 202 & & \\
\hline \multicolumn{9}{|c|}{ (b) ER- $\alpha_{1}-\alpha_{2}-\mathrm{SF}$ chains $\left({ }^{287} 114\right)$} \\
\hline $\begin{array}{c}E_{\mathrm{Lab}} \\
(\mathrm{MeV})\end{array}$ & Strip & $\begin{array}{c}E_{\mathrm{ER}} \\
(\mathrm{MeV})\end{array}$ & $\begin{array}{c}\delta t_{\text {ER- } \alpha} \\
\text { (s) }\end{array}$ & $\begin{array}{c}E_{\alpha 1} \\
(\mathrm{MeV})\end{array}$ & $\begin{array}{l}\delta t_{\alpha 1-\alpha 2} \\
\quad(\mathrm{~s})\end{array}$ & $\begin{array}{c}E_{\alpha 2} \\
(\mathrm{MeV})\end{array}$ & $\begin{array}{c}\delta t_{\alpha 2-\mathrm{SF}} \\
(\mathrm{s})\end{array}$ & $\begin{array}{c}E_{\mathrm{SF}} \\
(\mathrm{MeV})\end{array}$ \\
\hline \multirow[t]{5}{*}{235} & 11 & 10.2 & 0.599 & $10.00(7)$ & 3.782 & $8.94(7)$ & 0.830 & 134 \\
\hline & 2 & 6.6 & \multicolumn{2}{|c|}{ Missing $\alpha$} & 6.754 & $9.54(6)$ & 0.530 & 174 \\
\hline & 5 & 12.8 & 0.935 & $9.98(9)$ & 2.357 & 9.49(9) & 0.206 & 146 \\
\hline & 4 & 10.2 & 0.396 & $9.89(15)^{\mathrm{b}}$ & 2.808 & $9.53(6)$ & 0.0645 & 150 \\
\hline & 4 & 7.2 & & $\lg \alpha$ & 22.024 & $9.50(6)$ & 0.121 & 193 \\
\hline \multirow[t]{5}{*}{238} & 11 & 9.0 & 0.0985 & $10.06(7)$ & 1.706 & $9.7(2)^{\mathrm{b}}$ & 0.0495 & 200 \\
\hline & 4 & 11.9 & 1.829 & $9.99(6)$ & \multicolumn{2}{|c|}{ Missing $\alpha$} & 13.909 & $215^{\mathrm{c}}$ \\
\hline & 10 & 10.3 & 0.803 & $9.99(8)$ & 0.788 & $9.36(8)$ & 0.0750 & $213^{\mathrm{c}}$ \\
\hline & 8 & 8.8 & \multicolumn{2}{|c|}{ Missing $\alpha$} & 4.409 & $9.54(9)$ & 0.337 & 136 \\
\hline & 10 & 12.8 & 0.627 & $10.00(8)$ & 2.690 & $9.51(8)$ & 0.0637 & $201^{\mathrm{c}}$ \\
\hline \multirow[t]{6}{*}{244} & 9 & 7.3 & 0.199 & $10.09(45)^{\mathrm{a}}$ & 8.621 & $9.67(15)^{b}$ & 0.239 & $209^{c}$ \\
\hline & 2 & 10.4 & 0.0122 & $10.09(6)$ & 1.871 & $9.59(15)^{b}$ & 0.114 & $221^{c}$ \\
\hline & 2 & 10.1 & 0.400 & $10.31(48)^{\mathrm{a}}$ & 5.463 & $9.32(6)$ & 0.406 & 200 \\
\hline & 6 & 10.9 & 0.280 & $9.98(10)$ & 20.092 & $9.7(5)^{\mathrm{a}}$ & 0.418 & $216^{\mathrm{c}}$ \\
\hline & 1 & 12.0 & 0.276 & $10.02(6)$ & 7.877 & $9.50(45)^{\mathrm{a}}$ & \multicolumn{2}{|c|}{ Chain given in Fig. 4} \\
\hline & Mean values & & 0.538 & $10.02(6)$ & 5.278 & $9.53(6)$ & 0.267 & \\
\hline \multicolumn{9}{|c|}{ (c) ER- $\alpha /$ SF-SF chains $\left({ }^{286} 114\right)$} \\
\hline $\begin{array}{c}E_{\mathrm{Lab}} \\
(\mathrm{MeV})\end{array}$ & Strip & $\begin{array}{c}E_{\mathrm{ER}} \\
(\mathrm{MeV})\end{array}$ & $\begin{array}{c}\delta t_{\mathrm{ER}-\alpha / \mathrm{SF}} \\
(\mathrm{ms})\end{array}$ & $\begin{array}{c}E_{\alpha / \mathrm{SF}} \\
(\mathrm{MeV})\end{array}$ & $\begin{array}{c}\delta t_{\alpha-\mathrm{SF}} \\
(\mathrm{ms})\end{array}$ & $\begin{array}{c}E_{\mathrm{SF}} \\
(\mathrm{MeV})\end{array}$ & & \\
\hline \multirow[t]{7}{*}{244} & 1 & 10.9 & 14.2 & $10.10(15)^{b}$ & 0.978 & $213^{\mathrm{c}}$ & & \\
\hline & 3 & 11.5 & 181 & $201^{\mathrm{c}}$ & & & & \\
\hline & 6 & 13.0 & 84.7 & $10.25(10)$ & 0.458 & 189 & & \\
\hline & 3 & 12.9 & 135 & 162 & & & & \\
\hline & 4 & 10.3 & 185 & $10.20(6)$ & 1.586 & 164 & & \\
\hline & 7 & 13.3 & 41.4 & 218 & & & & \\
\hline & 5 & 8.9 & 335 & 182 & & & & \\
\hline \multirow[t]{3}{*}{250} & 6 & 10.5 & 603 & $228^{\mathrm{c}}$ & & & & \\
\hline & 4 & 13.3 & 150 & $10.34(22)^{b}$ & 0.143 & $211^{\mathrm{c}}$ & & \\
\hline & Mean values & & 192 & $10.21(6)$ & 0.791 & & & \\
\hline
\end{tabular}

${ }^{\mathrm{a}}$ Escaped $\alpha$ particle registered by side detector only.

${ }^{\mathrm{b}} \alpha$ particle registered by both focal-plane and side detectors.

${ }^{c}$ Fission event registered by both focal-plane and side detectors.

and that were followed by a beam-off SF event or one or more beam-off $\alpha$ particles with $E_{\alpha}=8.9-10 \mathrm{MeV}$ and a beam-on SF event in an elapsed time interval of up to $14 \mathrm{~min}$, without taking into account the position and strip number for the subsequent events. All of these $\alpha$ particles belong to the decays of ${ }^{286} 114$ and ${ }^{287} 114$.

The position deviations of the detected signals of recoil nuclei and subsequent sequential decays ( $\alpha$ and SF) for all observed decay chains originating from the mother nuclei ${ }^{286} 114$ and ${ }^{287} 114$ are shown in Fig. 3(a); they indicate a strong position correlation among the observed decays. In only three cases as positions of the chain members deviate from the average values by more than $1 \mathrm{~mm}$. These $\alpha$ particles were detected by both focal-plane and side detectors, but with low energies deposited in the focal plane of about $1.5,1.0$, and $4.2 \mathrm{MeV}$. The small amplitudes of these energy signals result in a degraded position resolution. The probability that all the observed events (including these three) are due to random detector background is very low, even for the experimental counting rates of events detected in beam, and 


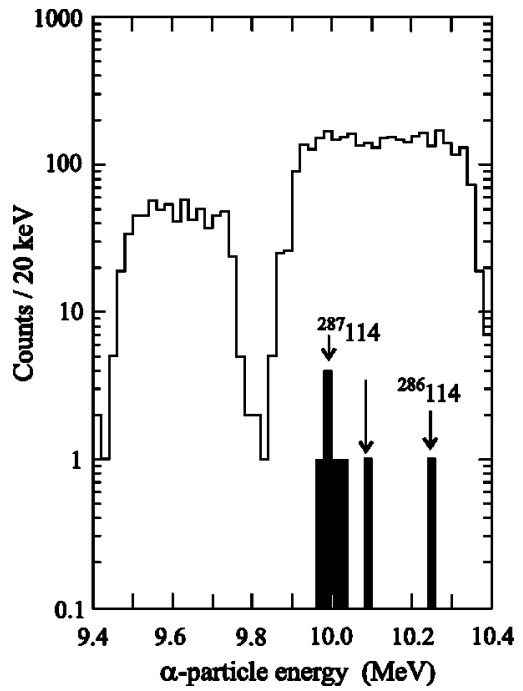

FIG. 2. Total $\alpha$-particle-like energy spectra of events that stopped the beam during the ${ }^{242} \mathrm{Pu}+{ }^{48} \mathrm{Ca}$ experiment. The solid histogram shows the energies of events that switched off the beam that were followed by a beam-off SF event or beam-off $\alpha$ particles with $E_{\alpha}=8.9-10 \mathrm{MeV}$ and a beam-on SF event, with a total elapsed time interval of up to $14 \mathrm{~min}$.

negligible for those decay chains registered during the beamoff period.

In the course of the experiments with the ${ }^{242} \mathrm{Pu}$ target, performed at four bombarding energies, 25 decay chains were detected that we assign to the decay of $Z=114$ nuclides. Some of the measured parameters of the members of the decay chains, namely, detector strip number, detected energies of events, and time intervals between events, are shown in Table II. The distribution of the 25 observed events over the strips is a consequence of the magnetic rigidity of the recoils in the gas-filled separator. The observed deflection corresponds to the separation of $Z=114$ recoils traversing the hydrogen media with an average ion charge of $5.9 \pm 0.2$ (compared with $q_{\text {calc }}=5.8$ ) [16]. The measured energies of the recoils also agree well with those expected from energystraggling and detector-performance calculations and with those measured previously in our ${ }^{244} \mathrm{Pu}+{ }^{48} \mathrm{Ca}$ experiments [4]. However, the radioactive decay characteristics of the observed recoils differ. This indicates that they originate from different $Z=114$ isotopes.

The decay chains can be sorted into three groups: decay chains of the ER- $\alpha-\alpha$-SF type lasting for $2-20 \mathrm{~s}$ (one special case is an ER- $\alpha-\alpha-\alpha-\alpha$-SF chain lasting for $6.5 \mathrm{~min}$ ) observed at beam energies $E_{\mathrm{L}}=235-244 \mathrm{MeV}$, listed in group (b) in Table II; shorter decays of the ER- $\alpha$-SF or ER-SF type spanning a typical time of 0.1-0.6 s, observed at higher beam energies $E_{\mathrm{L}}=244-250 \mathrm{MeV}$, and listed in group (c) in Table II; and, finally, a single ER- $\alpha$-SF event, $t \sim 4 \mathrm{~s}$, detected at the lowest beam energy $E_{\mathrm{L}}=235 \mathrm{MeV}$, and listed in group (a) in Table II.

In the ${ }^{242} \mathrm{Pu}+{ }^{48} \mathrm{Ca}$ reaction, we detected a total of $33 \alpha$ decays in the correlated decay chains shown in Table II. Four $\alpha$ particles are missing, which is entirely consistent with the $\alpha$-detection efficiency of the detector array that is approximately $87 \%$ of $4 \pi$. In Table II, such events are marked
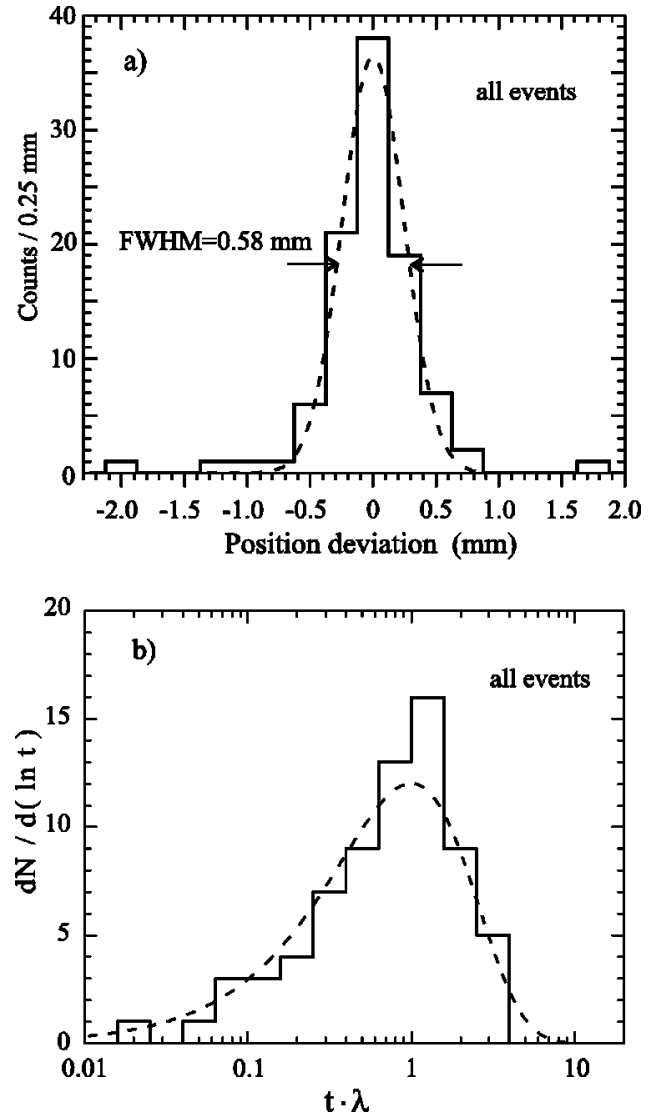

FIG. 3. (a) Relative position deviations of all events in the observed decay chains. (b) Relative time intervals of all events in the observed decay chains $\left(\lambda=\ln 2 / T_{1 / 2}\right)$ compared with the average half-lives assigned to the appropriate nuclides. Dashed lines are fits to the data.

"Missing $\alpha$." Their location within the decay chains of type ER- $\alpha_{1}-\alpha_{2}$-SF can be easily determined through comparison with the other 11 chains.

In the reaction ${ }^{238} \mathrm{U}+{ }^{48} \mathrm{Ca}$ (1070-h run), of the 31 detected decay signals with $E \geqslant 130 \mathrm{MeV}$, eight are assigned to the decays of the $Z=112$ isotopes (see Table III). In the series of ${ }^{238} \mathrm{U}+{ }^{48} \mathrm{Ca}$ irradiations, about 1740 beam-off intervals occurred, for a total of $31 \mathrm{~h}$. For this experiment, the $\alpha$-particle spectra measured during beam-on and beam-off intervals and the spectra of the fission fragments look practically the same as those measured in the reaction ${ }^{242} \mathrm{Pu}+{ }^{48} \mathrm{Ca}$ (see Fig. 1). In the $\alpha$ spectrum detected during those beam-off periods, we observed a single decay chain that we have determined to be ER- $\alpha-\alpha-\alpha-\alpha$-SF. An $\alpha$ particle preceding this chain has an energy $E_{\alpha 1}=9.56 \pm 0.08 \mathrm{MeV}$ (compare with the data shown in Fig. 4). This value coincides, within the energy resolution of the focal-plane detector, with the energy of $\alpha$ particles in other ER- $\alpha$-SF sequences observed at beam energy $E_{\mathrm{L}}$ $=234 \mathrm{MeV}$ when only SF events were detected during the beam off.

In the ${ }^{238} \mathrm{U}+{ }^{48} \mathrm{Ca}$ experiments, we detected eight decay sequences that we have assigned to the decay of $Z=112 \mathrm{nu}-$ clides. These can be separated into two types: ER- $\alpha$-SF chains spanning about $0.5-6 \mathrm{~s}$ that were observed at beam energies $E_{\mathrm{L}}=230-234 \mathrm{MeV}$, listed in group (a) in Table III; 
TABLE III. Decay chains observed in the ${ }^{238} \mathrm{U}+{ }^{48} \mathrm{Ca}$ reaction. Bold events were registered during a beam-off period. Time intervals for events following missing $\alpha$ were measured from preceding registered events and are shown in italic. The $\alpha$ particle energy errors are shown in parentheses.

\begin{tabular}{|c|c|c|c|c|c|c|}
\hline \multicolumn{7}{|c|}{ (a) ER- $\alpha$-SF chains $\left({ }^{283} 112\right)$} \\
\hline $\begin{array}{c}E_{\mathrm{Lab}} \\
(\mathrm{MeV})\end{array}$ & Strip & $\begin{array}{c}E_{\mathrm{ER}} \\
(\mathrm{MeV})\end{array}$ & $\begin{array}{c}\delta t_{\mathrm{ER}-\alpha} \\
(\mathrm{s})\end{array}$ & $\begin{array}{c}E_{\alpha} \\
(\mathrm{MeV})\end{array}$ & $\begin{array}{c}\delta t_{\alpha-\mathrm{SF}} \\
\quad(\mathrm{s})\end{array}$ & $\begin{array}{c}E_{\mathrm{SF}} \\
(\mathrm{MeV})\end{array}$ \\
\hline 230 & 3 & 13.0 & \multicolumn{2}{|c|}{ Missing $\alpha$} & 4.836 & 162 \\
\hline \multirow{7}{*}{234} & 5 & 13.0 & 5.155 & $9.56(8)$ & 0.0210 & 185 \\
\hline & 4 & 11.3 & \multicolumn{2}{|c|}{ Missing $\alpha$} & 0.776 & $186^{\mathrm{a}}$ \\
\hline & 5 & 7.3 & \multicolumn{2}{|c|}{ Missing $\alpha$} & 3.255 & 132 \\
\hline & 5 & 13.6 & 0.458 & $9.57(8)$ & 0.180 & 196 \\
\hline & 5 & 12.5 & 6.070 & $9.49(8)$ & 0.0317 & 194 \\
\hline & 5 & 11.3 & 8.746 & $9.56(8)$ & \multicolumn{2}{|c|}{ Chain given in Fig. 4} \\
\hline & Mean values & & 5.107 & $9.54(8)$ & 0.128 & \\
\hline \multicolumn{7}{|c|}{ (b) ER-SF chain $\left({ }^{282} 112\right)$} \\
\hline $\begin{array}{c}E_{\mathrm{Lab}} \\
(\mathrm{MeV})\end{array}$ & Strip & $\begin{array}{c}E_{\mathrm{ER}} \\
(\mathrm{MeV})\end{array}$ & $\begin{array}{c}\delta t_{\text {ER-SF }} \\
(\mathrm{ms})\end{array}$ & $\begin{array}{c}E_{\mathrm{SF}} \\
(\mathrm{MeV})\end{array}$ & & \\
\hline 240 & 6 & 10.8 & 0.207 & $222^{\mathrm{a}}$ & & \\
\hline
\end{tabular}

${ }^{\mathrm{a}}$ Fission event registered by both focal-plane and side detectors.

and shorter sequences, ER-SF, with $t_{\mathrm{SF}}<1 \mathrm{~ms}$ observed at $E_{\mathrm{L}}=240 \mathrm{MeV}$, listed in group (b) in Table III. Two longer ER- $\alpha_{1}-\alpha_{2}-\alpha_{3}-\alpha_{4}$-SF decay chains observed in the reaction ${ }^{242} \mathrm{Pu}+{ }^{48} \mathrm{Ca} \quad\left(E_{\mathrm{L}}=244 \mathrm{MeV}\right) \quad$ and $\quad{ }^{238} \mathrm{U}+{ }^{48} \mathrm{Ca} \quad\left(E_{\mathrm{L}}\right.$ $=234 \mathrm{MeV}$ ) are shown separately in Fig. 4. The distribution of the respective recoil nuclei over the detector strips corresponds to the separation of $Z=112$ evaporation residues produced in the reaction ${ }^{238} \mathrm{U}+{ }^{48} \mathrm{Ca}$ with $q_{\text {expt }}=6.1 \pm 0.2$ (compared with $\left.q_{\text {calc }}=5.9\right)$.

Characteristics of the decays in the observed chains, the average values of $E_{\alpha}, t_{\alpha}$, and $t_{\mathrm{SF}}$ determined from all the

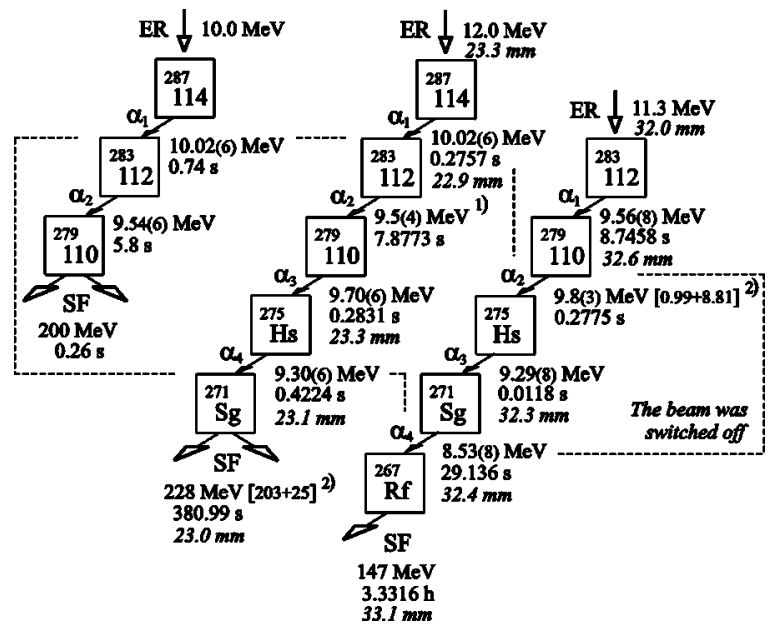

FIG. 4. Time sequences in the average decay chain of ${ }^{287} 114$ (left) and in selected decay chains observed in the ${ }^{242} \mathrm{Pu}+{ }^{48} \mathrm{Ca}$ (middle) and ${ }^{238} \mathrm{U}+{ }^{48} \mathrm{Ca}$ (right) reactions. Measured energies, time intervals, and positions of the observed decay events are shown. Energy uncertainties are shown in parentheses. (1) The energy of this event was detected by side detectors only. (2) The energies of events detected by both the focal-plane and side detectors, respectively, are shown in brackets. decay events of each type, are given in the bottom rows of Tables II and III. The production cross sections of the nuclides detected in our experiments, characterized by their types of decay, as a function of the excitation energies of the compound nuclei ${ }^{290} 114$ and ${ }^{286} 112$ are shown in Fig. 5. In addition, excitation functions of the reactions ${ }^{244} \mathrm{Pu}\left({ }^{48} \mathrm{Ca}, 3-5 n\right){ }^{287-289} 114$ that we measured earlier with the same experimental setup [4] and the presently available data for the reaction ${ }^{248} \mathrm{Cm}\left({ }^{48} \mathrm{Ca}, 3-4 n\right){ }^{292,293} 116$ are also shown, together with the Bass reaction barriers [17] and the calculated excitation functions [18] for all reactions. Comparing the decay properties of the observed nuclei (Tables II and III) and the excitation functions for their production (Fig. 5), we can deduce a consistent picture for the masses of the observed nuclides.

Decays of the daughter nuclei in the ER- $\alpha_{1}-\alpha_{2}$-SF chains observed in the reaction ${ }^{242} \mathrm{Pu}+{ }^{48} \mathrm{Ca}$ coincide in all the measured parameters $\left(E_{\alpha}, T_{\alpha}, T_{\mathrm{SF}}\right.$, and $\left.E_{\mathrm{SF}}\right)$ with the decay chain ER- $\alpha$-SF observed in the ${ }^{238} \mathrm{U}+{ }^{48} \mathrm{Ca}$ reaction. The maximum yields of the nuclides that undergo this type of decay correspond to the $3 n$-evaporation channel in fusion reactions ${ }^{242} \mathrm{Pu},{ }^{238} \mathrm{U}+{ }^{48} \mathrm{Ca}$. Therefore, the ER- $\alpha_{1}-\alpha_{2}$-SF decay chain from the reaction ${ }^{242} \mathrm{Pu}+{ }^{48} \mathrm{Ca}$ should be assigned to the decay of ${ }^{287} 114$. This conclusion is supported by the data from the reactions ${ }^{245} \mathrm{Cm},{ }^{244} \mathrm{Pu}+{ }^{48} \mathrm{Ca}$ in which similar decay chains were observed in $2 n$ - and $5 n$-evaporation channels [4], respectively. Accordingly, the ER- $\alpha$-SF chains observed in the reaction ${ }^{238} \mathrm{U}+{ }^{48} \mathrm{Ca}$ are due to the $\alpha$ decay of ${ }^{283} 112$ that is terminated by the spontaneous fission of the isotope ${ }^{279}$ Ds $\left(T_{\mathrm{SF}}=0.18 \mathrm{~s}\right)$.

The excitation functions and the decay properties of the shorter chain members (ER- $\alpha$-SF) detected in the ${ }^{242} \mathrm{Pu}$ $+{ }^{48} \mathrm{Ca}$ reaction, and ER-SF correlations in ${ }^{238} \mathrm{U}+{ }^{48} \mathrm{Ca}$ reaction, determine that these originate from the neighboring even-even isotopes ${ }^{286} 114$ and ${ }^{282} 112$, respectively. They were not observed in the reaction ${ }^{244} \mathrm{Pu}+{ }^{48} \mathrm{Ca}(6 n$ channel) 


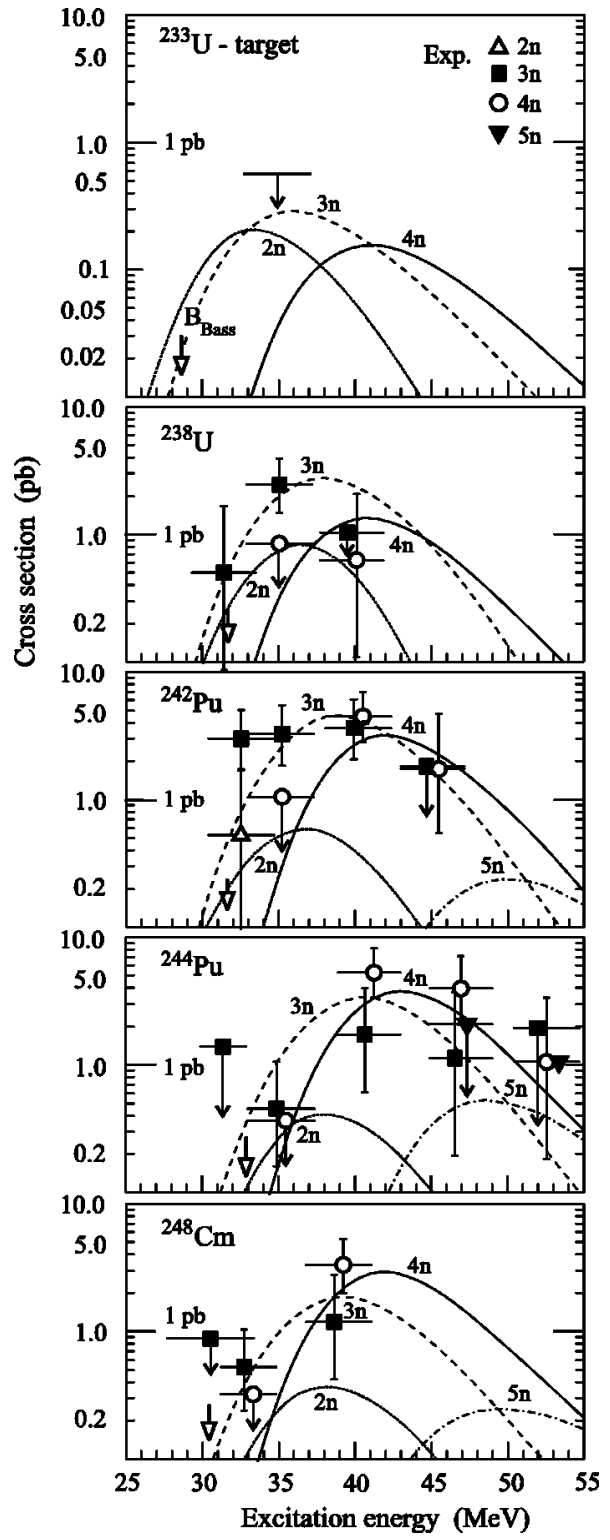

FIG. 5. Excitation functions for the $2 n(\triangle), 3 n(\square), 4 n(\bigcirc)$, and $5 n(\boldsymbol{\nabla})$ evaporation channels from the complete-fusion reactions ${ }^{233,238} \mathrm{U},{ }^{242,244} \mathrm{Pu},{ }^{248} \mathrm{Cm}+{ }^{48} \mathrm{Ca}$. The Bass barrier [17] is shown by an open arrow in each panel; in the topmost panel it is labeled with $B_{\text {Bass. }}$ Lines show the results of calculations [18]. Error bars correspond to statistical uncertainties.

but appear in the decays of ${ }^{290} 116$ and ${ }^{294} 118$ produced in the reactions ${ }^{245} \mathrm{Cm}\left({ }^{48} \mathrm{Ca}, 3 n\right){ }^{290} 116$ [4] and ${ }^{249} \mathrm{Cf}\left({ }^{48} \mathrm{Ca}, 3 n\right){ }^{294} 118[5]$, respectively. Note that in the decay of ${ }^{286} 114$, as observed in all of the experiments, only five $\alpha$ decays were observed out of 13 atoms $\left(b_{\alpha} \sim 0.4\right)$.

One more short-lived SF decay $\left(\Delta t=86 \mu \mathrm{s}, \quad E_{\mathrm{SF}}\right.$ $=149 \mathrm{MeV})$ was registered in the reaction ${ }^{238} \mathrm{U}+{ }^{48} \mathrm{Ca}$ at $E_{\mathrm{L}}$ $=234 \mathrm{MeV}$. Taking into account the measured transfer reaction cross sections with ${ }^{248} \mathrm{Cm}$ [19] and the yields of the fission isomers in heavy-ion reactions with ${ }^{238} \mathrm{U}[20]$, we cannot exclude that this SF decay was caused by the $21-\mu \mathrm{s}$ isomer ${ }^{241 \mathrm{mf}} \mathrm{Pu}[21]$. Because of the $85-\mu$ s dead time associated with the detection system for consecutive signals of the
recoil-SF type, decays of ${ }^{241 \mathrm{mf}} \mathrm{Pu}$ with shorter correlation times could not be detected.

Finally, a single ER- $\alpha$-SF event observed in the reaction ${ }^{242} \mathrm{Pu}+{ }^{48} \mathrm{Ca}$ at a beam energy $E_{\mathrm{L}}=235 \mathrm{MeV}$ agrees well in decay properties with the well-studied nuclide ${ }^{288} 114$ (12 events detected) that we previously synthesized in the reaction ${ }^{244} \mathrm{Pu}\left({ }^{48} \mathrm{Ca}, 4 n\right){ }^{288} 114$ [4]. This event should then be assigned to the decay of ${ }^{288} 114$ produced via $2 n$ evaporation with a cross section of about $0.5 \mathrm{pb}$.

As it can be seen in Fig. 5, the maximum cross section values that we measured for the reaction ${ }^{242} \mathrm{Pu}+{ }^{48} \mathrm{Ca}$ at the energy $E^{*}=40.2 \mathrm{MeV}$ are $\sigma_{3 n}=3.6_{-1.7}^{+3.4} \mathrm{pb}$ and $\sigma_{4 n}$ $=4.5_{-1.9}^{+3.6} \mathrm{pb}$; for the reaction ${ }^{238} \mathrm{U}\left({ }^{48} \mathrm{Ca}, 3 n\right)^{283} 112$ the maximum values are $\sigma_{3 n}=2.5_{-1.1}^{+1.8} \mathrm{pb}$ at $E^{*}=35.0 \mathrm{MeV}$ and $\sigma_{4 n}$ $=0.6_{-0.5}^{-1.6} \mathrm{pb}$ at $E^{*}=39.8 \mathrm{MeV}$. In view of the present results, the non-observation of decay chains of element 112 isotopes in the reaction of ${ }^{238} \mathrm{U}$ with $231-\mathrm{MeV}^{48} \mathrm{Ca}$ projectiles $\left(E^{*}\right.$ $=32.5 \mathrm{MeV}$ ) at LBNL [22] is obviously due to insufficient experimental sensitivity. The "upper cross section limit for one event" set in the recent LBNL experiment $(1.6 \mathrm{pb})$ corresponds to a statistically valid $84 \%$ upper limit of $2.9 \mathrm{pb}$ [23] provided all the parameters of the experiment were chosen optimally.

We have also studied the reaction ${ }^{233} \mathrm{U}+{ }^{48} \mathrm{Ca}$ at $E_{\mathrm{L}}$ $=240 \mathrm{MeV}$; despite an accumulated beam dose of about 8 $\times 10^{18}$ ions, we did not observe any decay chains that could be attributed to the decay of isotopes of element 112. This non-observation could be associated with either of two reasons: (1) Either the lifetime of ${ }^{278} 112$ is less than the time of flight of the recoils from the target to detectors $(\sim 1 \mu \mathrm{s})$ and the dead time of the detection system ( $6 \mu$ s for ER- $\alpha$ signals); or (2) its production cross section is below the sensitivity of the present experiment. The first hypothesis is not realistic: an even-even nuclide ${ }^{278} 112$ with a half-life of $\leqslant 7 \mu$ s should $\alpha$-decay with an energy $Q_{\alpha} \geqslant 11.9 \mathrm{MeV}$; this significantly deviates from the experimental values of $Q_{\alpha}$ observed in the decays of the neighboring isotopes of element 112; see Fig. 6. Furthermore, the descendant nuclei of ${ }^{278} 112\left({ }^{274} \mathrm{Ds},{ }^{270} \mathrm{Hs},{ }^{266} \mathrm{Sg},{ }^{262} \mathrm{Rf}[10,11]\right)$ were not observed. For the partial half-life for the SF decay of ${ }^{278} 112$, one would expect a larger $T_{\mathrm{SF}}$ than that observed for ${ }^{282} 112$ due to a closer proximity to the deformed shell $N=162$ [28]. The even-odd isotopes ${ }^{277} 112$ and ${ }^{279} 112$ (products of the $2 n$ - and $4 n$-evaporation channels) should have $T_{\alpha}$ values exceeding the dead time of the detection system [e.g., $\left.T_{1 / 2}{ }^{277} 112\right)$ $=0.6 \mathrm{~ms}[8,9]]$. We take this as support for the second assumption, and we calculate an upper cross section limit of $\sigma_{2-4 n} \leqslant 0.6 \mathrm{pb}$ for the reaction ${ }^{233} \mathrm{U}\left({ }^{48} \mathrm{Ca}, 2-4 n\right){ }^{277-279} 112$ at $E_{\mathrm{L}}=240 \mathrm{MeV}$.

\section{DISCUSSION}

\section{A. Nuclear properties}

Now that the mass numbers of the observed $Z=112,114$, and 116 isotopes have been deduced on the basis of measured excitation functions, let us analyze specific properties of the decay chains of the isotopes synthesized in the present work. In the ER- $\alpha_{1}-\alpha_{2}$-SF chains arising in the decay of the 
isotope ${ }^{287} 114$, the energy of the first $\alpha$ particle is $E_{\alpha 1}$ $=10.02 \pm 0.06 \mathrm{MeV}$. In all 12 events in which the decay of the mother nuclide has been observed, the measured values of $E_{\alpha 1}$ agree with the given value within the detector resolution characteristic of the actual detection conditions, as well as with the value measured for this isotope in [4]. The loss of the first $\alpha$ particle in three events does not influence our interpretation of the decay scenario of this nuclide. The energy of the second $\alpha$ particle in 11 cases of 14 is $E_{\alpha 2}$ $=9.54 \pm 0.06 \mathrm{MeV}$. This value, as we noted above, agrees well with the four measured $\alpha$ energies of ${ }^{283} 112$ (see Table III), produced as a fusion-evaporation product in the reaction ${ }^{238} \mathrm{U}\left({ }^{48} \mathrm{Ca}, 3 n\right){ }^{283} 112$, and with energies registered for this isotope in previous experiments [4].

However, three of the measured energies of the second alpha, $E_{\alpha 2}=8.94(7), 9.36(8)$, and 9.32(6) MeV, are different enough from the average value of $E_{\alpha 2}$ that they are beyond the experimental uncertainties of measuring $\alpha$ energies associated with the focal-plane detector. This means that the observed $\alpha$ decays of ${ }^{283} 112$ correspond to transitions to various excited states in the daughter nucleus ${ }^{279} 110$. Given the accuracy with which we measure the $\alpha$-particle energies (which varies depending on experimental conditions) and the relatively low statistics, we can evaluate the probability of such transitions as being about $20 \%$. In principle, this kind of decay pattern for an odd nucleus is likely since ${ }^{279} 110(N$ $=169$ ) is located in an intermediate region seven neutrons above the deformed shell closure at $N=162$ and 15 neutrons below the spherical shell at $N=184$. Finally, in Table II, we present a single case in which the second $\alpha$ particle is missing and the spontaneous fission is detected $13.9 \mathrm{~s}$ after emission of the first $\alpha$ particle. Since the half-life of ${ }^{279} 110$ determined from 21 events is $0.18 \mathrm{~s}$, the observed time interval $t_{\text {ER-SF }}$ is therefore defined either by the time of emission of the unobserved second $\alpha$ particle or by the spontaneous fission of ${ }^{283} 112$ occurring with a probability of $\leqslant 10 \%$. As a whole, the decay properties of the isotope ${ }^{283} 112$ produced in the reactions ${ }^{238} \mathrm{U}+{ }^{48} \mathrm{Ca}$ and ${ }^{242} \mathrm{Pu}+{ }^{48} \mathrm{Ca}$ do not depend on whether it is observed as a primary nucleus or as an $\alpha$-decay product of a preceding mother nucleus.

In the reaction ${ }^{242} \mathrm{Pu}+{ }^{48} \mathrm{Ca}$, we observed no decay chains of the ER- $\alpha$-SF type of 3-5 min in duration that had been reported from previous experiments performed with the VASSILISSA separator $[6,7]$. It should be noted that while the reported values [7] of $T_{\alpha}=5.5_{-2}^{+10} \mathrm{~s}$ deduced from two events and $E_{\alpha}=10.29 \pm 0.02 \mathrm{MeV}$ measured for a single event are similar to those observed for ${ }^{287} 114$ in our experiments, the half-life of the daughter nucleus differs by an order of magnitude [6,7]. The existence of such a long-lived decay mode for the even-odd nucleus ${ }^{283} 112$ reported previously $[6,7]$ cannot be entirely excluded; however, the present data indicate that its probability does not exceed $10 \%$.

At the same time, in one of the 15 decays of ${ }^{287} 114$ produced in the reaction ${ }^{242} \mathrm{Pu}+{ }^{48} \mathrm{Ca}$ and in one of the seven decays of ${ }^{283} 112$ produced in the reaction ${ }^{238} \mathrm{U}+{ }^{48} \mathrm{Ca}$, we observed lengthy sequential $\alpha$ decays that were terminated by $\mathrm{SF}$ with long lifetimes: $t_{\mathrm{SF}} \sim 6.3 \mathrm{~min}$ and $3.3 \mathrm{~h}$, respectively; see Fig. 4. These rare decays result from $\alpha / \mathrm{SF}$ competition in the decay of ${ }^{279} 110\left(b_{\alpha} \sim 10 \%\right.$, including three decays observed in [4]) and end in the spontaneous fission of the neutron-rich isotopes ${ }^{271} \mathrm{Sg}(N=165)$ and ${ }^{267} \mathrm{Rf}(N$ $=163$ ). Comparing the two decay chains given in Fig. 4, one can see that ${ }^{271} \mathrm{Sg}$ undergoes both $\alpha$ decay $\left(E_{\alpha}=8.53 \mathrm{MeV}\right)$ and SF. The increased stability of ${ }^{267} \mathrm{Rf}$ and ${ }^{271} \mathrm{Sg}$ with respect to SF is due to their proximity to the deformed shell at $N=162$.

In four of the observed nine events from the reaction ${ }^{242} \mathrm{Pu}+{ }^{48} \mathrm{Ca}$ (in five of the observed 13 decays total, including the results of $[4,5]$ ), the even-even isotope ${ }^{286} 114$ undergoes $\alpha$ decay with $E_{\alpha}=10.20 \mathrm{MeV}, b_{\alpha} \sim 40 \%$, in competition with SF. Its $\alpha$-decay daughter, ${ }^{282} 112$, decays by SF with a short half-life, $T_{\mathrm{SF}}=0.5 \mathrm{~ms}$.

Because of low statistics, we cannot rule out the possibility that several similar half-life values contribute to the decays of each of the observed isotopes of elements 112 and 114. Time distributions, presented in sum in Fig. 3(b), allow us to characterize each decay type by a single half-life value, bearing in mind the actual statistical uncertainties.

Data on the decay characteristics of the isotopes 286,287114 , ${ }^{282,283} 112$, and ${ }^{279} 110$, as well as ${ }^{275} \mathrm{Hs},{ }^{271} \mathrm{Sg}$, and ${ }^{267} \mathrm{Rf}$ synthesized in the reactions ${ }^{242} \mathrm{Pu},{ }^{238} \mathrm{U}+{ }^{48} \mathrm{Ca}$, are summarized in Table IV. Included also are the data for heavier isotopes with $Z=110-114$ that we produced earlier in the reaction ${ }^{244} \mathrm{Pu}+{ }^{48} \mathrm{Ca}[1,4]$ and the isotopes of heavier elements with $Z=116,118$ from our experiments ${ }^{245,248} \mathrm{Cm},{ }^{249} \mathrm{Cf}+{ }^{48} \mathrm{Ca}[1,2,4,5,29]$. The decay modes, number of observed events used to determine the decay properties, and values of $T_{1 / 2}, E_{\alpha}$, and $Q_{\alpha}$ are shown in Table IV. Expected values of $T_{\alpha}$ were calculated from the measured $Q_{\alpha}$ from the Geiger-Nuttall relationship using the Viola-Seaborg formula [30]: $\log _{10}\left(T_{\alpha}\right)=(a Z+b) * Q_{\alpha}{ }^{-1 / 2}+c Z+d$. Parameters were fitted to the $T_{\alpha}$ vs $Q_{\alpha}$ values of 65 previously known even-even nuclei with $Z>82$ and $N>126(a=1.787, b=$ $-21.40, c=-0.2549, d=-28.42)$. The limiting values of $Q_{\alpha}$ for the spontaneously fissioning nuclei were estimated in the same way.

Experimental $\alpha$-decay energies of the isotopes with $Z$ $=100-118$, together with the decay energies of the same nuclides calculated in the macroscopic-microscopic (MM) nuclear model [24,25], are compared in Fig. 6. The agreement between theory and experiment becomes somewhat worse as one moves from the deformed nuclei in the vicinity of neutron shells $N=152$ and 162 to the more neutron-rich nuclides with $N \geqslant 169$. In this region, experimentally measured values of $Q_{\alpha}$ are less than the values calculated from the model by $\leqslant 0.5 \mathrm{MeV}$. In general, this is good agreement, considering that these theoretical predictions of the MM model are matched over a broad unexplored nuclear domain by the measured $Q_{\alpha}$ values for 14 nuclides with $Z$ $=106-118$ and $N=165-177$. One should note that the predictions of other models within the Skyrme-Hartree-FockBogoliubov (SHFB) [31] and the relativistic mean field (RMF) [32] methods also compare well with the experimental results. These models predict the same spherical neutron shell at $N=184$, but different proton shells, $Z=114$ (MM) and $Z=120,124$, or 126 (SHFB, RMF), yet all describe the experimental data equally well. Such insensitivity with respect to the various models can be explained either by the 
TABLE IV. Decay properties of nuclei produced in this work and $[1,2,4,5]$.

\begin{tabular}{|c|c|c|c|c|c|c|c|}
\hline$Z$ & $A$ & $\begin{array}{c}\text { No. } \\
\text { observed }^{\text {a }}\end{array}$ & $\begin{array}{c}\text { Decay mode, } \\
\text { branch }^{\text {b }}\end{array}$ & Half-life $^{c}$ & $\begin{array}{l}\text { Expected } \\
\text { half-life }\end{array}$ & $E_{\alpha}(\mathrm{MeV})$ & $Q_{\alpha}(\mathrm{MeV})$ \\
\hline 118 & 294 & $1 / 1$ & $\alpha$ & $1.8_{-1.3}^{+75} \mathrm{~ms}$ & $0.4 \mathrm{~ms}$ & $11.65 \pm 0.06$ & $11.81 \pm 0.06$ \\
\hline \multirow[t]{4}{*}{116} & 293 & $3 / 3$ & $\alpha$ & $53_{-19}^{+62} \mathrm{~ms}$ & $80 \mathrm{~ms}$ & $10.53 \pm 0.06$ & $10.67 \pm 0.06$ \\
\hline & 292 & $4 / 5$ & $\alpha$ & $18_{-6}^{+16} \mathrm{~ms}$ & $40 \mathrm{~ms}$ & $10.66 \pm 0.07$ & $10.80 \pm 0.07$ \\
\hline & 291 & $2 / 2$ & $\alpha$ & $6.3_{-2.5}^{+11.6} \mathrm{~ms}$ & $20 \mathrm{~ms}$ & $10.74 \pm 0.07$ & $10.89 \pm 0.07$ \\
\hline & 290 & $2 / 2$ & $\alpha$ & $15_{-6}^{+26} \mathrm{~ms}$ & $10 \mathrm{~ms}$ & $10.85 \pm 0.08$ & $11.00 \pm 0.08$ \\
\hline \multirow[t]{4}{*}{114} & 289 & $8 / 8$ & $\alpha$ & $2.7_{-0.7}^{+1.4} \mathrm{~s}$ & $2 \mathrm{~s}$ & $9.82 \pm 0.06$ & $9.96 \pm 0.06$ \\
\hline & 288 & $12 / 11$ & $\alpha$ & $0.80_{-0.18}^{+0.32} \mathrm{~s}$ & $0.8 \mathrm{~s}$ & $9.95 \pm 0.07$ & $10.09 \pm 0.07$ \\
\hline & 287 & $15 / 15$ & $\alpha$ & $0.51_{-0.10}^{+0.18} \mathrm{~s}$ & $0.5 \mathrm{~s}$ & $10.02 \pm 0.06$ & $10.16 \pm 0.06$ \\
\hline & 286 & $11 / 5$ & $\begin{array}{c}\alpha: 0.4 \\
\mathrm{SF}: 0.6\end{array}$ & $0.16_{-0.03}^{+0.07} \mathrm{~s}$ & $0.2 \mathrm{~s}$ & $10.20 \pm 0.06$ & $10.35 \pm 0.06$ \\
\hline \multirow[t]{4}{*}{112} & 285 & $8 / 8$ & $\alpha$ & $34_{-9}^{+17} \mathrm{~s}$ & $50 \mathrm{~s}$ & $9.16 \pm 0.06$ & $9.29 \pm 0.06$ \\
\hline & 284 & 12 & SF & $101_{-22}^{+41} \mathrm{~ms}$ & & & $\leqslant 9.85$ \\
\hline & 283 & $18 / 18$ & $\begin{array}{c}\alpha: 1 \\
\mathrm{SF}: \leqslant 0.1\end{array}$ & $4.0_{-0.7}^{+1.3} \mathrm{~s}$ & $3 \mathrm{~s}$ & $9.54 \pm 0.06$ & $9.67 \pm 0.06$ \\
\hline & 282 & 6 & SF & $0.50_{-0.14}^{+0.33} \mathrm{~ms}$ & & & $\leqslant 10.82$ \\
\hline \multirow[t]{2}{*}{110} & 281 & 8 & $\mathrm{SF}$ & $9.6_{-2.5}^{+5.0} \mathrm{~s}$ & & & $\leqslant 9.05$ \\
\hline & 279 & $21 / 2$ & $\begin{array}{c}\alpha: 0.1 \\
\text { SF: } 0.9\end{array}$ & $0.18_{-0.03}^{+0.05} \mathrm{~s}$ & $0.2 \mathrm{~s}$ & $9.70 \pm 0.06$ & $9.84 \pm 0.06$ \\
\hline 108 & 275 & $2 / 2$ & $\alpha$ & $0.15_{-0.06}^{+0.27} \mathrm{~s}$ & $0.8 \mathrm{~s}$ & $9.30 \pm 0.07$ & $9.44 \pm 0.07$ \\
\hline 106 & 271 & $2 / 1$ & $\begin{array}{c}\alpha: 0.5 \\
\text { SF: } 0.5\end{array}$ & $2.4_{-1.0}^{+4.3} \mathrm{~min}$ & $0.8 \mathrm{~min}$ & $8.53 \pm 0.08$ & $8.65 \pm 0.08$ \\
\hline 104 & 267 & 1 & SF & $2.3_{-1.7}^{+98} \mathrm{~h}$ & & & $\leqslant 8.22$ \\
\hline
\end{tabular}

${ }^{\mathrm{a}}$ Number of events used for calculations of half-lives/ $\alpha$-particle energies, respectively.

${ }^{\mathrm{b}}$ Branching ratio is not shown if only one decay mode was observed.

${ }^{\mathrm{c}}$ Error bars correspond to $68 \%$ confidence level if more than one event was observed, for only one registered event, the error bars correspond to $95 \%$.

remoteness of nuclei under consideration from the closed shell at $N=184$ or by the relatively weaker influence of the proton shells at $Z=114$ or higher, compared with that of the neutron shell at $N=184$.

Of particular interest for comparisons with theory are the long decay chains like those shown in Fig. 4. The decay properties of the isotopes of element 114 are generally determined by the spherical shells $Z=114$ and $N=184$. According to $\mathrm{MM}$ model calculations [25], the nucleus ${ }^{287} 114$ is almost spherical $\left(\beta_{2}=0.088\right)$. In a succession of sequential $\alpha$ decays, the descendant nuclei move away from the closed $N$ $=184$ shell and approach the deformed shell at $N=162$. The terminating nucleus, ${ }^{267} \mathrm{Rf} \quad(N=163)$, is deformed $\left(\beta_{2}\right.$ 0.23) [25]. Accordingly, it is possible to compare the predicted changes in the structure of nuclei in the decay chain originating with the decay of ${ }^{287} 114$ with the experimental data. As can be seen in Fig. 6, for the last nuclei in the decay chain $\left({ }^{275} \mathrm{Hs} \rightarrow{ }^{271} \mathrm{Sg} \rightarrow{ }^{267} \mathrm{Rf}\right)$, experimental values of $Q_{\alpha}$ practically coincide with calculations; here one observes a considerable decrease of $Q_{\alpha}$ due to the proximity of the neutron shell at $N=162$. In the decay chain ${ }^{291} 116 \rightarrow \cdots$ $\rightarrow{ }^{267} \mathrm{Rf}$ (see Fig. 6), we observed a similar variation in $\alpha$-decay energies as we reported for decay chains starting with ${ }^{287} 115$ or ${ }^{288} 115$ [15]. The slope of $Q_{\alpha}$ vs neutron number remains practically the same for elements $112-116$ but increases significantly for the nuclides with $Z=111$ and 110 . Such an effect might be caused by the transition from spherical nuclear shapes to deformed shapes during successive $\alpha$ decays, in agreement with MM calculations [25].

Unfortunately, in the decay of the even-odd nucleus ${ }^{287} 114$, longer decay chains are blocked by the spontaneous fission of ${ }^{279} 110\left(b_{\alpha} \sim 10 \%\right)$. It is partially for this reason that we attempted to synthesize the odd-odd nucleus ${ }^{288} 115$ in the reaction ${ }^{243} \mathrm{Am}+{ }^{48} \mathrm{Ca}$. The result was a lengthy decay chain consisting of five $\alpha$ decays and terminated by SF [15], which is the principal decay mode.

With the decay properties of the $Z=110-114$ nuclides presented in Table IV and Fig. 6, we can revisit the longlived ER- $\alpha$ - $\alpha$ - $\alpha$-SF decay chain detected in the reaction ${ }^{244} \mathrm{Pu}+{ }^{48} \mathrm{Ca}$ in 1998 [33] at $E^{*}=35 \mathrm{MeV}$ that was considered to be a candidate for the decay originating from ${ }^{289} 114$. The experimental values of $E_{\alpha}$ and $t_{\alpha}$ for the three $\alpha$ decays, as well as the limiting value of $E_{\alpha}$ for the final SF nucleus agree with expectations for the nuclides in the chain of sequential $\alpha$ decays of the isotope ${ }^{290} 114$. The observed production cross section of this nuclide $(\sim 0.2 \mathrm{pb})$ is consistent with $\sigma_{2 n} \sim 0.5 \mathrm{pb}$ observed in the reaction ${ }^{242} \mathrm{Pu}\left({ }^{48} \mathrm{Ca}, 2 n\right){ }^{288} 114$ which has a $2.3-\mathrm{MeV}$ higher $Q$ value than does the ${ }^{244} \mathrm{Pu}+{ }^{48} \mathrm{Ca}$ system. However, one has to suppose a considerable increase in stability against decay by SF 

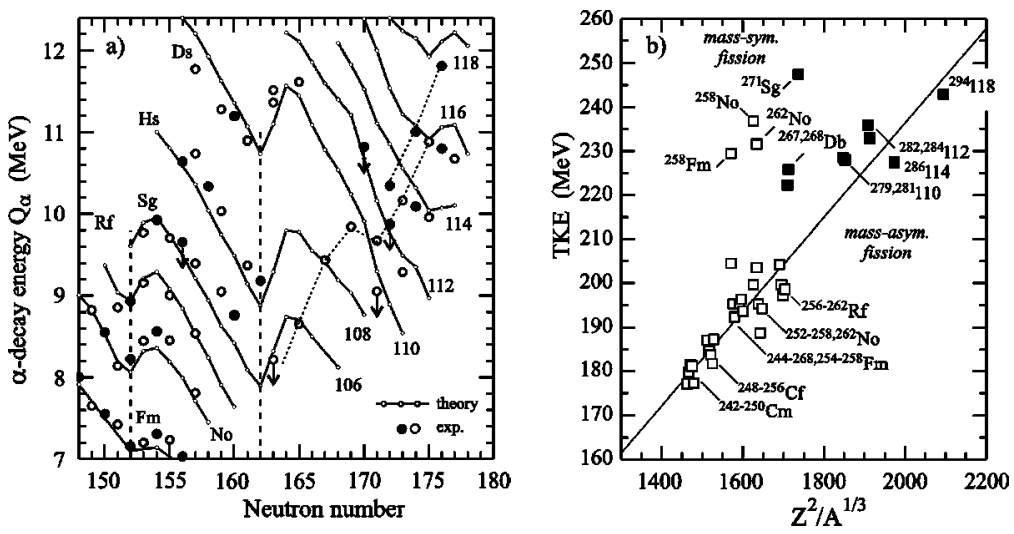

FIG. 6. (a) $\alpha$-decay energy vs neutron number for isotopes of even- $Z$ elements with $Z \geqslant 100$ (solid circles, even-even isotopes; open circles, even-odd isotopes) [8-11,21]. Data at $N \geqslant 163$ that are connected by dashed lines are from [1,2,4,5] and the present work. Solid lines show the theoretical $Q_{\alpha}$ values [24,25] for even- $Z=100-118$ elements. (b) Experimental values of TKE vs $Z^{2} / A^{1 / 3}$ (previously measured data from [26] and references therein), open squares; experimental data from the present work, solid squares. The solid line is the linear fit to the data, excluding the mass-symmetric fissioners [27].

of ${ }^{286} 112$ and ${ }^{282} 110$ compared with their lighter neighbors for $\alpha$ decay to dominate. On the other hand, such a long chain could originate from a rare decay branch of the evenodd nuclide ${ }^{289} 114$ starting from an isomeric state [31].

All the decay chains presented in Tables II and III end in spontaneous fission. In cases when the detector array registered both fission fragments, their sum energy $E_{\text {tot }}$ could be used to determine the total kinetic energy of the fission fragments. The value of TKE for all the SF isotopes obtained in the ${ }^{48} \mathrm{Ca}$-induced reactions given in Table IV is plotted in Fig. 6(b), together with the previously known data for the isotopes with $Z \geqslant 96$ (see Ref. [26] and references therein). It is observed that, with the transition $Z \geqslant 110$, the TKE increases with increasing $Z$ in agreement with the previously established dependence of TKE vs $Z^{2} / A^{1 / 3}$, typical for the asymmetric fission of lighter nuclei. We speculate that this is caused by the influence of the spherical shells $Z=50$ and $N$ $=82$ on the formation of the light fission fragment in the scission of superheavy nuclei $[3,26,34]$. In the spontaneous fission of the lighter nuclides ${ }^{267} \mathrm{Db},{ }^{268} \mathrm{Db}$, and ${ }^{271} \mathrm{Sg}$ with $N=162,163$, and 165 , the same effect apparently results in symmetric fission with high kinetic energy, characteristic of the close scission configuration that takes place in the bimodal fission of the heavy isotopes of Fm, Md, and No [26,34].

\section{B. Cross sections for the $x \boldsymbol{n}$-evaporation channels in the reactions $\mathrm{U}, \mathrm{Pu}+{ }^{48} \mathrm{Ca}$}

Considering the production of evaporation residues in the complete fusion of actinide target nuclei with ${ }^{48} \mathrm{Ca}$ within the classical concept of formation of a compound nucleus, one can express the cross section of the various neutron evaporation channels as $\sigma_{x n}\left(E^{*}\right) \sim P_{\mathrm{CN}}\left(E^{*}\right) \times W_{\text {surv }}\left(E^{*}\right)$. Here, $P_{\mathrm{CN}}$ and $W_{\text {surv }}$ are the probability of the formation of compound nucleus and the survival probability in the deexcitation to the ground state by emission of neutrons and $\gamma$ rays, respectively, as a function of excitation energy $E^{*}$. Accordingly, the value of $P_{\mathrm{CN}}$ is determined by the probability of capture, $P_{\text {capt }}$, and the dynamics of motion of the compos- ite system from an initial state of two contacting nuclei to the compact, almost spherical shape of the compound nucleus. In this process, which is characterized by a substantial change of nuclear deformation, the nuclear system can separate into two fragments (undergo so-called quasifission), having not reached the equilibrium deformation; thus, $P_{\mathrm{CN}}$ $\ll P_{\text {capt }}[18]$. In such a situation, the probability $P_{\mathrm{CN}}$ depends substantially on the primary conditions that determine the trajectory of the collective motion of the heavy nucleus. A strong Coulomb repulsion of nuclei at the primary reaction stage also decreases $P_{\mathrm{CN}}$ in favor of deep inelastic reactions. Both hindrances, for different reasons, considerably limit the formation of the compound nuclei.

Another stage of the process is the deexcitation of the compound nucleus via sequential neutron emission. In statistical models, for heavy nuclei, the survival probability is determined mainly by the relation of partial widths $\left(\Gamma_{n} / \Gamma_{\text {tot }}\right) \approx\left(\Gamma_{n} / \Gamma_{f}\right): W_{\text {surv }}\left(E^{*}\right) \sim \prod_{i=1}^{x}\left(\Gamma_{n} / \Gamma_{f}\right)_{i} \sim \prod_{i=1}^{x} \exp \left[\left(B_{f}\right.\right.$ $\left.\left.-B_{n}\right) / T\right]_{i}$. Here $B_{f}$ and $B_{n}$ are fission barrier and neutron separation energy in the compound nucleus, respectively, with excitation energy $E^{*}$ and temperature $T$, and $x$ is the number of emitted neutrons.

For the reactions under consideration, leading to nuclei with $Z_{\mathrm{CN}}=112-114$ and $N_{\mathrm{CN}}=169-178$, nuclear masses calculated in various models give an uncertainty of neutron binding energy $\Delta B_{n} \leqslant 1 \mathrm{MeV} \quad[24,25,31,32,35]$. Modeldependent variations in the fission barrier height $B_{f}$ are much larger, $\Delta B_{f} \approx 3-4 \mathrm{MeV}[24,35,36]$. It should be noted that with increasing nuclear temperature, the amplitude of the shell correction decreases, which results in a decrease of $B_{f}$ (see, e.g., [37]). This effect is especially important for the heavy nuclei with $Z_{\mathrm{CN}} \geqslant 100$ for which the fission barrier height is completely determined by shell effect.

The same calculational method that was developed earlier for describing the cross sections for the production of No isotopes in the reactions ${ }^{204,206-208} \mathrm{~Pb}+{ }^{48} \mathrm{Ca}$ [38], was employed to reproduce the measured values of $\sigma_{x n}\left(E^{*}\right)$ presented in Fig. 5. These calculations (not shown in the figure) disagreed with the experiment; calculated maxima of the excitation functions of the $2 n$ and $3 n$ channels were shifted to 

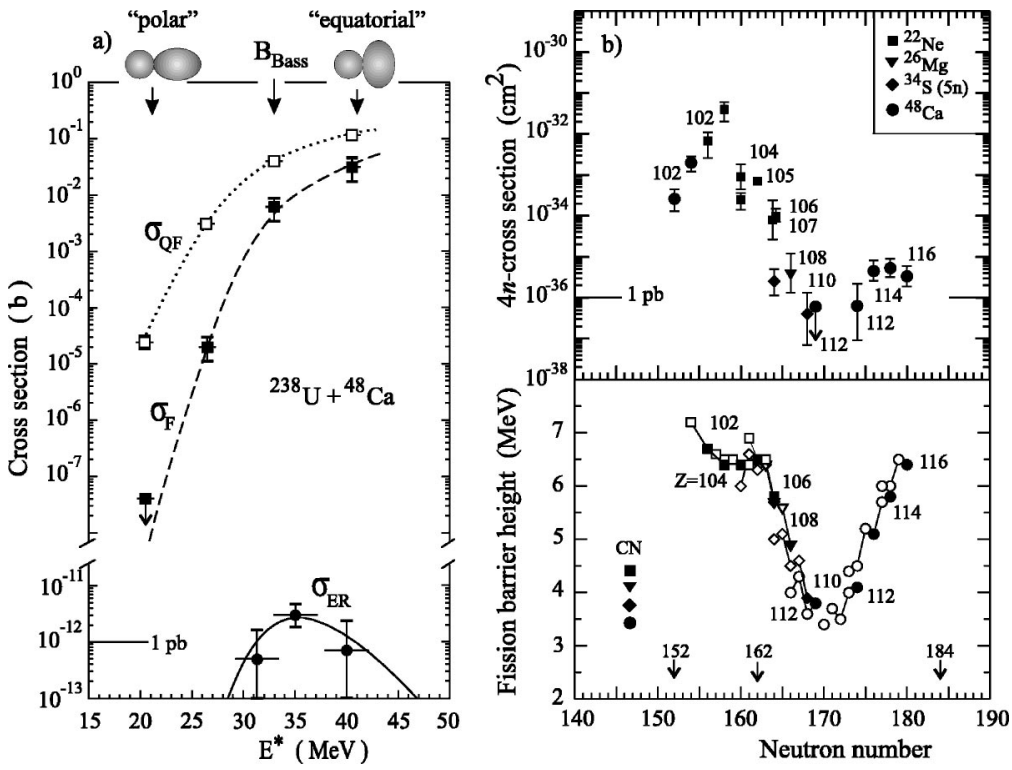

FIG. 7. (a) Comparison of the cross section as a function of excitation energy $E^{*}$ for quasifission $\left(\sigma_{\mathrm{QF}}\right)$, fission $\left(\sigma_{\mathrm{F}}\right)$, and ER $\left(\sigma_{\mathrm{ER}}\right)$ for the ${ }^{238} \mathrm{U}+{ }^{48} \mathrm{Ca}$ reaction. Experimental data are from [3] and present work, and the lines show calculations [18]. Note the location of the "polar" and "equatorial" touching configurations with respect to the Bass barrier, and the discontinuous cross section scale. (b) Comparison of hot fusion cross sections for the production of $Z \geqslant 102$ nuclides using a variety of heavy-ion beams (top panel). Note the relatively constant cross sections for $Z \geqslant 112$ nuclides produced with ${ }^{48}$ Ca projectiles. We show the fission barrier heights as a function of neutron number on the bottom panel [28,36] (lower panel). Solid symbols correspond to the number of neutrons in compound nuclei formed in different reactions. Note the influence of the closed shells-especially the spherical shell closure at $N=184$. There is a correspondence between the increased barrier height in the lower panel and the enhanced cross sections of ER production in ${ }^{48} \mathrm{Ca}$-induced reactions in the top panel at the same neutron numbers.

lower energies and the modeled cross sections $\sigma_{\mathrm{ER}}$ were considerably higher than those experimentally observed. Variation of the statistical model parameters in calculations of the survival probabilities of compound nuclei with $Z=112$ and 114 did not eliminate these discrepancies.

One should note that all of the events observed in the production of isotopes of elements 112 and 114 with the fusion reactions ${ }^{238} \mathrm{U},{ }^{242,244} \mathrm{Pu}+{ }^{48} \mathrm{Ca}$ were detected at energies above the Coulomb barrier, (Fig. 5). In contrast, in cold fusion reactions of the target nuclei of ${ }^{208} \mathrm{~Pb}$ and ${ }^{209} \mathrm{Bi}$ with more massive projectiles $(A \geqslant 48)$, the maximum yield of evaporation residues is observed at subbarrier projectile energies $[8,9]$.

The observed shift of excitation functions to higher energies cannot be explained by the dynamic limitations of fusion, because in our case we use more asymmetric reactions than are encountered in cold fusion reactions. The effect may be associated with the static deformation of the target nuclei, whose orientation at the point of collision leads to various configurations of the composite system at the very start of its path toward the spherical shape of the compound nucleus. "Equatorial" collisions, characterized by minimum distances between the centers of the interacting nuclei, correspond to the most compact configurations of the composite system. For such collisions, the Coulomb barrier of the reaction ${ }^{242,244} \mathrm{Pu}+{ }^{48} \mathrm{Ca}$ is about $20 \mathrm{MeV}$ higher than is that encountered in "polar" collisions, which are characterized by the maximum distance between reaction partners, in which the heavy nucleus is formed with maximum deformation (Fig. 7). All of the other configurations are intermediate between these extremes. If the angle of impact corresponds to a more compact configuration, then the compound nucleus has a higher probability of reaching the final spherical shape $[18,39]$, and such collisions will be decisive for determining the fusion-evaporation cross section $\sigma_{\mathrm{ER}}=\Sigma \sigma_{x n}$.

The quasifission reaction channels that are characterized by the formation of fragments with highly asymmetric mass division most probably occur at the earliest stage of collective motion, when the compound nucleus is still strongly deformed. The principal contribution to the quasifission cross section at lower excitation energies $\left(E^{*} \leqslant 30 \mathrm{MeV}\right)$, is from the "polar" interactions of the colliding nuclei [18]. Therefore, the dependence $\sigma_{\mathrm{QF}}\left(E^{*}\right) \approx \sigma_{\text {capt }}\left(E^{*}\right)$ is relatively weak up to $E^{*} \approx 27 \mathrm{MeV}$; see Fig. 7. In contrast, the complete fusion of the nucleus that arises from the more compact configuration is more likely to undergo more symmetric fission. The observed dependence $\sigma_{\mathrm{F}}\left(E^{*}\right)$ is steeper than that of $\sigma_{\mathrm{QF}}\left(E^{*}\right)$ and is shifted to higher values of $E^{*}$. Here, the competing quasifission channel limits the contribution of "polar" collisions to the formation of a compound nucleus. If we suggest that $\sigma_{\mathrm{CN}}\left(E^{*}\right)$ corresponds to a some more strict selection of the target nucleus orientation compared with $\sigma_{\mathrm{F}}\left(E^{*}\right)$ (the additional change of distance between centers of nuclei spans over only $4 \%$ ), the statistical calculations reproduce well the experimental dependences $\sigma_{\mathrm{ER}}\left(E^{*}\right)$ for all the reactions presented in Fig. 5 [18].

Let us further consider the production cross sections of the evaporation residues. From numerous experiments it is known that in the synthesis of heavy nuclei with $Z \geqslant 102$, both in hot and cold fusion reactions, the cross section $\sigma_{\mathrm{ER}}$ 
decreases rapidly with increasing $Z_{\mathrm{CN}}$. Extrapolating the dependence $\sigma_{\mathrm{ER}}\left(Z_{\mathrm{CN}}\right)$ to $Z>110$, we would arrive at extremely low cross sections for the production of isotopes of element $114\left(\sigma_{\mathrm{ER}} \sim 1-10 \mathrm{fb}\right)$. However, experimental values of $\sigma_{\mathrm{ER}}\left(Z_{\mathrm{CN}}=114\right)$ observed in the reactions ${ }^{242,244} \mathrm{Pu}+{ }^{48} \mathrm{Ca}$ appeared to be about three orders of magnitude higher. We consider that this fundamental discrepancy has a firm physical explanation.

In cold fusion reactions, the decrease of $\sigma_{\mathrm{ER}}$ with increasing $Z_{\mathrm{CN}}$ is associated with the dynamic hindrances of the fusion of massive nuclei [18]. Here, extrapolation to superheavy nuclei appears to be justified, as the hindrances should increase with increasing mass and nuclear charge of the projectile. In asymmetric hot fusion reactions using light projectile nuclei, like the isotopes of $\mathrm{C}, \mathrm{O}, \mathrm{Ne}, \mathrm{Mg}$, there are practically no fusion limitations. Here, the decrease of $\sigma_{\mathrm{ER}}$ for higher values of $Z_{\mathrm{CN}}$ is determined by the decreasing survivability of the compound nuclei. As shown above, the value of $W_{\text {surv }}$ strongly depends on $\left(B_{\mathrm{f}}-B_{\mathrm{n}}\right)$. Since the value of $B_{\mathrm{f}}$ is completely determined by the amplitude of the shell correction $\left(B_{\mathrm{f}}^{\mathrm{LD}} \approx 0\right.$ for the nuclei with $\left.Z \geqslant 102\right)$, it strongly depends on the neutron number of the compound nucleus. The high fission barriers and correspondingly high cross sections $\sigma_{\mathrm{ER}}$ observed in the synthesis of elements with $Z$ $=102-106$ are associated with the significant shell effects at $N=152$ and at $N=162$ [Fig. 7(b)]. For heavier nuclei with $N>162, B_{\mathrm{f}}$ values decrease until the next spherical shell at $N=184$ starts influencing the fission barrier, while $B_{\mathrm{n}}$ values steadily decrease in this region with increasing neutron number. Upon approaching the closed neutron shell, the fission barriers will increase again, according to all microscopic models (see, e.g., $[35,36]$ ). That should result in a substantial increase of $\sigma_{\mathrm{ER}}$.

Thus, if the theoretical predictions of the existence of closed nuclear shells in the domain of superheavy elements are correct, they should be characterized not only by higher stability to various decay modes (longer half-lives) but also by a relatively high probability of production. As we have shown in Fig. 7(b), the increase of the height of the fission barrier due to the influence of the shell closure at $N=184$ is expected only for the neutron-rich nuclei with $N>170$. For these nuclei, an increase of neutron number in the compound nucleus results in an increase of the production cross section observed in the experiments. We consider this to be the major advantage of using the complete fusion reactions involving the neutron-rich transuranic target nuclei and the ${ }^{48} \mathrm{Ca}$ projectile for the synthesis of superheavy elements.

Indeed, the experimental data show that for the nuclei with $Z=112$ and 114 and $N_{\mathrm{CN}}=174-178$ the cross section of the $4 n$-evaporation channel (an "open" channel, well above the fusion barrier) systematically increases with increasing neutron number and reaches the maximum value of about $5 \mathrm{pb}$ in the reaction ${ }^{244} \mathrm{Pu}+{ }^{48} \mathrm{Ca}$. The low cross section for the formation of the isotope ${ }^{278} 112$ in the reaction ${ }^{233} \mathrm{U}$ $+{ }^{48} \mathrm{Ca}$ with $N_{\mathrm{CN}}=169$ has the corresponding explanation. Additionally, in the reaction ${ }^{248} \mathrm{Cm}+{ }^{48} \mathrm{Ca}\left(Z_{\mathrm{CN}}=116\right.$ and $N_{\mathrm{CN}}=180$ ), one could expect a higher $\sigma_{4 n}$ cross section; however, the excitation function is not yet complete. The cross section of the reaction ${ }^{248} \mathrm{Cm}\left({ }^{48} \mathrm{Ca}, 4 n\right){ }^{292} 116$ at $E^{*}$
$=38.9 \mathrm{MeV}$ (about $3 \mathrm{MeV}$ below the expected maximum cross section for the $4 n$ channel) has already reached the value $3.3_{-1.4}^{+2.5} \mathrm{pb}$. In this reaction, six decay chains of the new isotope ${ }^{292} 116$ were observed. The decay properties of ${ }^{292} 116$ are also included in Table IV.

\section{CONCLUSION}

Several conclusions follow from the above analysis and discussion of the present experimental data. We measured production cross sections and studied radioactive decay properties of the isotopes ${ }^{282,283} 112,{ }^{286,287} 114$, and ${ }^{292,293} 116$ synthesized in complete fusion reactions with ${ }^{48} \mathrm{Ca}$ projectiles. The mass numbers of the synthesized isotopes were determined from excitation functions of the $3 n$ - and $4 n$-evaporation channels of the reactions ${ }^{238} \mathrm{U},{ }^{242} \mathrm{Pu}$, and ${ }^{248} \mathrm{Cm}+{ }^{48} \mathrm{Ca}$ and $3 n-5 n$ channels of the reaction ${ }^{244} \mathrm{Pu}$ $+{ }^{48} \mathrm{Ca}[4]$.

The even-odd isotope ${ }^{287} 114$ undergoes mostly sequential $\alpha$ - $\alpha$-SF decay with a typical total decay time of about $2-20 \mathrm{~s}$. The total decay time is most strongly influenced by the relatively long-lived daughter nuclide ${ }^{283} 112\left(T_{\alpha}=4 \mathrm{~s}\right)$, which should be regarded as the longest-lived element 112 isotope for experiments aimed at the investigation of the chemical properties of element 112 in the reaction ${ }^{238} \mathrm{U}$ $+{ }^{48} \mathrm{Ca}$.

The SF nuclide ${ }^{279} 110$ undergoes $\alpha$ decay with a probability of $\sim 10 \%$, and the chain of subsequent decays is terminated by the SF of either ${ }^{271} \mathrm{Sg}$ or ${ }^{267} \mathrm{Rf}$. Their long lifetimes of minutes and hours are caused by the influence of the deformed shells at $Z=108$ and at $N=162$.

The isotope ${ }^{286} 114$ demonstrates an $\alpha$-SF decay pattern. The probability of spontaneous fission of the even-even nucleus ${ }^{286} 114$ is about $60 \%$; its $\alpha$-decay daughter, ${ }^{282} 112$, decays by SF with a short half-life $\left(T_{\mathrm{SF}}=0.5 \mathrm{~ms}\right)$.

The observed decay properties of the four isotopes of element 114 with masses 286-289 provide a consistent mass identification for all of the heavier even- $Z$ nuclei, in particular, the three isotopes of element 116 with masses 290, 291, and 293 that we synthesized earlier in the reactions ${ }^{245,248} \mathrm{Cm}+{ }^{48} \mathrm{Ca}[2,4]$, and also the isotope of element 118 with $A=294$ we observed as an $\alpha-\alpha$-SF chain in the reaction ${ }^{249} \mathrm{Cf}+{ }^{48} \mathrm{Ca}$ [5]. They define also the masses of neutron-rich nuclei with $Z=104-108$ that appear in the decay chains of the mother nucleus ${ }^{287} 114$.

The decay properties of nuclei in the decay chain ${ }^{290} 116(\alpha) \rightarrow{ }^{286} 114(\alpha / \mathrm{SF}) \rightarrow{ }^{282} 112(\mathrm{SF}), \quad$ synthesized individually in the three reactions ${ }^{245} \mathrm{Cm},{ }^{242} \mathrm{Pu},{ }^{238} \mathrm{U}+{ }^{48} \mathrm{Ca}$, coincide well with those measured for the descendant nuclei of the heavy even-even nuclide ${ }^{294} 118$ produced in the reaction ${ }^{249} \mathrm{Cf}\left({ }^{48} \mathrm{Ca}, 3 n\right){ }^{294} 118$ at $E^{*}=29.2 \mathrm{MeV}$.

All the observed $\alpha$-decay sequences end in SF characterized by a high TKE of the fragments. For superheavy nuclei with $Z \geqslant 110$, the value of TKE increases with $Z$ following the dependence of TKE vs $Z^{2} / A^{1 / 3}$ expected for asymmetric fission. Spontaneous fission of the nuclei with $Z=106$ and $N=165$, as well as ${ }^{267,268} \mathrm{Db}(N=162,163)$ with an abnormally high kinetic energy release is most probably associated with the symmetric fission decay mode. 
The ER production cross sections in the complete fusion reactions with ${ }^{48} \mathrm{Ca}$ are determined by the survivability of nuclei and depend mostly on their fission barrier height. The expected increase of fission barrier heights on approaching the neutron shell at $N=184$ leads to an increase in the evaporation residue cross section; a conclusion supported by our experimental data. Thus, the effect of nuclear shells in the domain of superheavy elements results not only in substantially higher stability to various decay modes, but also in an increase of the production cross section for complete fusion reactions with ${ }^{48} \mathrm{Ca}$ projectiles. The observed 3-5 MeV upward shift of the maxima of the evaporation residue yields in the reactions of actinide nuclei with ${ }^{48} \mathrm{Ca}$ projectiles with respect to the calculated Coulomb barrier for spherical nuclei can be attributed to the selection of the entrance states associated with the orientation of the deformed target nuclei in collisions with ${ }^{48} \mathrm{Ca}$.

The present experimental data on the production cross sections and decay properties of even- $Z$ nuclides can be used for determining the conditions for synthesis and prediction of the decay properties of odd- $Z$ nuclides. Our interpretation of the data of the experiments on the synthesis of element 115 in the reactions ${ }^{243} \mathrm{Am}\left({ }^{48} \mathrm{Ca}, 3-4 n\right){ }^{288,287} 115[15]$ is in agreement with the results of the present work.

\section{ACKNOWLEDGMENTS}

This work has been performed with the support of the Russian Ministry of Atomic Energy and RFBR Grant No. 04-02-17186. The ${ }^{233} \mathrm{U}$ and ${ }^{242} \mathrm{Pu}$ target material were provided by RFNC-VNIIEF, Sarov, Russia. The ${ }^{248} \mathrm{Cm}$ target material was provided by the U.S. DOE through ORNL. Much of the support for the LLNL authors was provided through the U.S. DOE under Contract No. W-7405-Eng-48. These studies were performed in the framework of the Russian Federation/U.S. Joint Coordinating Committee for Research on Fundamental Properties of Matter.
[1] Yu. Ts. Oganessian et al., Phys. Rev. C 62, 041604(R) (2000); Phys. At. Nucl. 63, 1679 (2000).

[2] Yu. Ts. Oganessian et al., Phys. Rev. C 63, 011301(R) (2001); Phys. At. Nucl. 64, 1349 (2001); Eur. Phys. J. A 15, 201 (2002).

[3] M. G. Itkis et al., in Proceedings of International Workshop on Fusion Dynamics at the Extremes, Dubna, Russia, 2000, edited by Yu. Ts. Oganessian and V. I. Zagrebaev (World Scientific, Singapore, 2001), p. 93; J. Nucl. Radiochem. Sci. (Japan) 3, 57 (2002).

[4] Yu. Ts. Oganessian et al., Phys. Rev. C 69, 054607 (2004); Nucl. Phys. A734, 109 (2004).

[5] Yu. Ts. Oganessian et al., JINR Communication D7-2002-287, 2002; Lawrence Livermore National Laboratory Report UCRL-ID-151619, 2003.

[6] Yu. Ts. Oganessian et al., Eur. Phys. J. A 5, 63 (1999); 19, 3 (2004).

[7] Yu. Ts. Oganessian et al., Nature (London) 400, 242 (1999).

[8] S. Hofmann and G. Münzenberg, Rev. Mod. Phys. 72, 733 (2000).

[9] S. Hofmann et al., Eur. Phys. J. A 14, 147 (2002); Z. Phys. A 354, 229 (1996).

[10] Ch. E. Düllmann et al., Nature (London) 418, 859 (2002).

[11] Yu. A. Lazarev et al., Phys. Rev. Lett. 73, 624 (1994); A. Türler et al., Phys. Rev. C 57, 1648 (1998); Eur. Phys. J. A 15, 271 (2002).

[12] Yu. Ts. Oganessian et al., in Proceedings of the Fourth International Conference on Dynamical Aspects of Nuclear Fission, Častá-Papiernička, Slovak Republic, 1998 (World Scientific, Singapore, 2000), p. 334; K. Subotic et al., Nucl. Instrum. Methods Phys. Res. A 481, 71 (2002).

[13] W. D. Myers and W. J. Swiatecki, Nucl. Phys. A601, 141 (1996)

[14] F. Hubert, R. Bimbot, and H. Gauvin, At. Data Nucl. Data Tables 46, 1 (1990); L. C. Northcliffe and R. F. Schilling, Nucl. Data, Sect. A 7, 233 (1970).
[15] Yu. Ts. Oganessian et al., Phys. Rev. C 69, 021601 (2004).

[16] Yu. Ts. Oganessian et al., Phys. Rev. C 64, 064309 (2001).

[17] R. Bass, in Proceedings of the Symposium on Deep Inelastic and Fusion Reactions with Heavy Ions, West Berlin, 1979, edited by W. von Oertzen, Lecture Notes in Physics Vol. 117 (Springer-Verlag, Berlin, 1980), p. 281.

[18] V. I. Zagrebaev, M. G. Itkis, and Yu. Ts. Oganessian, Phys. At. Nucl. 66, 1033 (2003); V. I. Zagrebaev, in Tours Symposium on Nuclear Physics, edited by M. Arnould, M. Lewitowicz, G. Münzenberg, H. Akimune, M. Ohta, H. Utsunomiya, T. Wada, and T. Yamagata, AIP Conf. Proc. No. 704 (AIP, Melville, NY, 2004), p. 31.

[19] D. Lee et al., Phys. Rev. C 25, 286 (1982); D. C. Hoffman et al., ibid. 31, 1763 (1985).

[20] S. M. Polikanov et al., Zh. Eksp. Teor. Fiz. 42, 1464 (1962) [Sov. Phys. JETP 15, 1016 (1962)]; Yu. V. Lobanov et al., Yad. Fiz. 1, 67 (1965).

[21] Table of Isotopes, 8th ed., edited by R. B. Firestone and V. S. Shirley (Wiley, New York, 1996).

[22] W. Loveland et al., Phys. Rev. C 66, 044617 (2002).

[23] K.-H. Schmidt et al., Z. Phys. A 316, 19 (1984).

[24] R. Smolańczuk and A. Sobiczewski, in Proceedings of XV Nuclear Physics Divisional Conference "Low Energy Nuclear Dynamics," St. Petersburg, Russia, 1995 (World Scientific, Singapore, 1995), p. 313; R. Smolańczuk, Phys. Rev. C 56, 812 (1997).

[25] I. Muntian et al., Acta Phys. Pol. B 34, 2073 (2003); Phys. At. Nucl. 66, 1015 (2003).

[26] D. C. Hoffman and M. R. Lane, Radiochim. Acta 70/71, 135 (1995).

[27] V. E. Viola Jr., Nucl. Data, Sect. A 1, 391 (1966).

[28] R. Smolańczuk, J. Skalski, and A. Sobiczewski, Phys. Rev. C 52, 1871 (1995).

[29] Yu. Ts. Oganessian et al. (unpublished).

[30] V. E. Viola Jr., and G. T. Seaborg, J. Inorg. Nucl. Chem. 28, 741 (1966). 
[31] S. Ćwiok, W. Nazarewicz, and P. H. Heenen, Phys. Rev. Lett. 83, 1108 (1999); J. F. Berger, D. Hirata, and M. Girod, Acta Phys. Pol. B 34, 1909 (2003); S. Typel and B. A. Brown, Phys. Rev. C 67, 034313 (2003).

[32] M. Bender, Phys. Rev. C 61, 031302 (2000); P.-G. Reinhard et al., in Tours Symposium on Nuclear Physics IV, edited by M. Arnould, M. Lewitowicz, Yu. Ts. Oganessian, H. Akimune, M. Ohta, H. Utsunomiya, T. Wada, and T. Yamagata, AIP Conf. Proc. No. 562 (AIP, Melville, NY, 2001), p. 377; Z. Ren, Phys. Rev. C 65, 051304(R) (2002).

[33] Yu. Ts. Oganessian et al., Phys. Rev. Lett. 83, 3154 (1999).
[34] E. K. Hulet et al., Phys. Rev. C 40, 770 (1989).

[35] P. Möller et al., At. Data Nucl. Data Tables 59, 185 (1995); A. Mamdouh et al., Nucl. Phys. A679, 337 (2001); T. Bürvenich et al., Phys. Rev. C 69, 014307 (2004).

[36] I. Muntian, Z. Patyk, and A. Sobiczewski, Acta Phys. Pol. B 34, 2141 (2003).

[37] Z. Lojewski, V. V. Pashkevich, and S. Cwiok, Nucl. Phys. A436, 499 (1985).

[38] Yu. Ts. Oganessian et al., Phys. Rev. C 64, 054606 (2001).

[39] A. Iwamoto, P. Möller, J. R. Nix, and H. Sagawa, Nucl. Phys. A596, 329 (1996). 